\title{
Field to Field coupled Fluid Structure Interaction: A Reduced Order Model study
}

\author{
Alexis Tello ${ }^{1}$, Ramon Codina ${ }^{1,2}$ \\ ${ }^{1}$ Universitat Politècnica de Catalunya (UPC), Jordi Girona 1-3, 08034 Barcelona, Spain \\ ${ }^{2}$ International Centre for Numerical Methods in Engineering (CIMNE), C/ Gran Capità S/N, 08034 Barcelona, Spain
}

\begin{abstract}
SUMMARY
The standard Fluid-Structure Interaction (FSI) coupling, that uses as unknowns velocity and pressure for the fluid and displacements for the solid, is compared against two novel types of coupling, the first one a three-field coupling (velocity-pressure-stress/displacement-pressure-stress) introduced by the authors in a recent work, and a two-field coupling (velocity-pressure/displacement-pressure) introduced in this paper, in this way completing our set of Field to Field (F2F) equations, all stabilized by means of the Variational Multi-Scale (VMS) method using dynamic and orthogonal sub-scales. The solid two-field FSI coupling formulation is benchmarked statically and dynamically. Proper Orthogonal Decomposition (POD) is applied to all three FSI formulations to obtain reduced basis and asses their performance in a reduced space. Numerical tests are shown comparing all three formulations. By correctly resolving the Cauchy stress tensor, the three-field FSI coupling proves to provide more accurate results in both Full Order Model (FOM) and Reduced Order Model (ROM) spaces than its counterparts for a similar number of degrees of freedom, making it a reliable formulation. $[\mathrm{F} 2 \mathrm{~F} /$ pairing appears to be beneficial, providing more accurate results in all cases shown; mixed pairing with a three-field formulation in the solid appears to produce very precise results as well. Copyright (c) 0000 John Wiley \& Sons, Ltd.
\end{abstract}

Received ...

KEY WORDS: Fluid Structure Interaction (FSI); Mixed Methods; Field to field (F2F) coupling; Reduced Order Models (ROM); Proper Orthogonal Decomposition (POD); Variational MultiScale Method (VMS); Dynamic sub-grid scales; Orthogonal Sub-grid Scales (OSS);

\section{INTRODUCTION}

The term 'Field to Field (F2F) coupling' is inspired from our work on Mixed Methods in the research of Fluid-Structure Interaction (FSI) problems to express the coupling between two domains by means of the same set of unknowns, see [29]. The idea was developed foreseeing its applicability in the field of Reduced Order Models (ROM) with the aim to further improve the accuracy of the solution in the reduced space. As it was seen in [30], [FSI ROM] standard coupling requires an important portion of the reduced basis to produce meaningful results. Later in [29] the benefit of F2F coupling is seen in the form of improved convergence, increased precision and less vulnerability to time-step change and mesh coarsening. In this work we explore the possible benefits that come with dealing with matching degrees of freedom for a reduced order problem.

${ }^{*}$ Correspondence to: Corresponding author ramon.codina@upc.edu (R. Codina)

Copyright (c) 0000 John Wiley \& Sons, Ltd.

Prepared using nmeauth.cls [Version: 2010/05/13 v3.00] 
FSI is a topic that can be treated in many ways, each with its benefits and drawbacks. As in previous works, we deal with a conforming formulation where the mesh reproduces the solution domains, solid and fluid, coupled through an interface on which a Dirichlet-Neumann iterative scheme is designed, converging to the strongly coupled problem. This kind of coupling is generally affected by long solution times and convergence difficulty on the interface boundaries, although it has benefits, such as the modularity of the solvers (for the solid and for the fluid) and the possibility of independent and efficient scaling and preconditioning. In the context of ROM, there is also the possibility to choose between a purely monolithic approach, as in [2, 3], or a partitioned scheme iterated to achieve strong coupling, as in [31, 18, 33]; this last option is adopted in some applications and large scale calculations, as in [20] or the pioneering work [21], in which a complete aircraft is modeled. It is not the purpose of this work to design efficient iteration-by-subdomain schemes, and thus we restrict ourselves to the Dirichlet-Neumann coupling. Yet, the stabilized formulation we employ alleviates considerably the number of iterations required to converge to the coupled solution. For more details on FSI coupling schemes and how ROM] is applied, see [30].

For all the formulations for each field (solid or fluid) to be described, we use the Variational MultiScale (VMS) approach to design stabilized formulations [15, 10], assuming that the Full Order Model (FOM) is a finite element (FE) method. As a distinctive feature of this work, we use the same VMS formulation for the ROM as for the FOM, and idea introduced in [24] and later expanded in [22]. This formulation uses dynamic and orthogonal sub-scales (see [8, 12]), properties that we found crucial for the success of our approach. Other attempts to employ VMS ideas in the ROM context can be found in [1, 16, 28].

The present work aims to expand on the research done in [30, 29]. First we introduce a simplification of the three-field formulation proposed in [29] for the solid so that instead of resolving for displacement-stress-pressure we solve for displacement-pressure and leave the stress as a postprocess. Notice that in this work we will use the term stress when talking about the deviatoric stress, not to be confused with the full stress tensor; more details about this can be found in Section 2.1. This displacement-pressure formulation is well known in linear elasticity, and in particular it is required if purely incompressible solids need to be modeled. Here we develop it in the context of finite strain elasticity using an updated Lagrangian description and stabilized with the same VMS approach as in [30]. This new formulation will help us to complete our F2F|FSI] equations so that we will be able to accurately benchmark and analyze them in the FOM ROM space. The focus is in the application of the different kind of FSI couplings to the field of reduced order modeling and in the evaluation of the importance of a F2F pairing in comparison with the standard FSI scheme, which uses a velocity-pressure formulation for the fluid and an irreducible displacement formulation for the solid.

The paper first deals with the solid two-field formulation in Section 2. The F2F strategy in FSI is then described in Section 3, where the options we consider are described. The ROM approach we employ is summarized in Section 4. The numerical schemes presented are then tested in Section 5. Some final conclusions close the paper in Section 6.

\section{TWO FIELD ELASTO-DYNAMIC SOLID EQUATIONS}

In this section we present the two-field elasto-dynamic solid equations, their manipulation to obtain the governing equations and the way we approximate them using our VMS formulation, the main features being the use of orthogonal and dynamic sub-scales arising from the VMS concept. Such a formulation for finite strain elasticity in an updated Lagrangian framework is developed here for the first time. The three-field formulation has been presented in [29]; we will follow closely the notation and development presented there, adapted now to the two-field formulation. 
The dynamic conservation of momentum equation for the solid problem can be written as:

$$
\left.\rho_{\mathrm{sl}} \partial_{t t} d_{i}-\partial_{j} \sigma_{i j}=\rho_{\mathrm{sl}} f_{\mathrm{sl}, i} \quad \text { in } \Omega_{s}, t \in\right] 0, t_{\mathrm{f}}[,
$$

where $\Omega_{\mathrm{sl}}$ is the domain of $\mathbb{R}^{n_{\mathrm{d}}}$ defined by the movement of the solid during the time interval $\left[0, t_{\mathrm{f}}\right]$, $n_{\mathrm{d}}=2$ or 3 is the number of space dimensions, $\partial_{t}$ denotes the partial time derivative (and thus $\partial_{t t}$ is the second derivative with respect to time), and $\partial_{i}$ the derivative with respect to the $i$ th Cartesian coordinate $x_{i}, i=1, \ldots, n_{\mathrm{d}}$. The unknowns for this problem are the displacement field and the Cauchy stress tensor, with Cartesian components $d_{i}$ and $\sigma_{i j}$, respectively, $i, j=1, \ldots, n_{\mathrm{d}}$. In this work vectors and tensors are assumed to be represented by their Cartesian components. In particular, $f_{\mathrm{sl}, i}$ is the vector of external forces acting on the solid, its density being $\rho_{\mathrm{sl}}$. Finally, in Eq. (1) and below repeated indexes imply summation over the number of space dimensions.

Eq. (1) will be expressed in an updated Lagrangian reference system for which the stress tensor has to be given in spatial form, this is, in terms of the Cauchy stress $\sigma_{i j}$. If the expression of the stress is defined in terms of the displacement and inserted into Eq. (1), we will call irreducible the resulting problem posed for $d_{i}$ alone, if the stress is expressed in terms of the deviatoric stress $s_{\mathrm{sl}, i j}$ and the pressure $p_{\mathrm{sl}}$ we will call this a three-field formulation, see [29]. Finally if the stress is expressed in terms of the pressure $p_{\mathrm{sl}}$ and the displacements we will call this a two-field formulation. Initial and boundary conditions have to be appended to this equation.

\subsection{Split of the stress tensor and field equations}

We can define the pressure and the deviatoric stress of the stress tensor in the following way:

$$
\begin{gathered}
p_{\mathrm{sl}}=\frac{1}{n_{\mathrm{d}}} \sigma_{k k}, \\
s_{\mathrm{sl}, i j}=\sigma_{i j}-p_{\mathrm{sl}} \delta_{i j},
\end{gathered}
$$

where $\delta_{i j}$ is Kronecker's delta. If we assume the material model to be Neo-Hookean, the stress tensor is given by:

$$
\sigma_{i j}=\frac{1}{J}\left[\left(\lambda \ln (J)-\mu_{\mathrm{sl}}\right) \delta_{i j}+\mu_{\mathrm{sl}} b_{i j}\right],
$$

where $J$ is the determinant of the displacement gradient $F_{i J}, \lambda$ and $\mu_{\mathrm{sl}}$ are Lame's parameters, $b_{i j}$ is the left Cauchy tensor and $b_{i i}$ its trace. These are defined as follows:

$$
J=\operatorname{det}\left(F_{i J}\right), \quad F_{i J}=\frac{\partial x_{i}}{\partial X_{J}}, \quad b_{i j}=F_{i K} F_{j K},
$$

where $x_{i}$ are the coordinates defined in the spatial frame of reference and $X_{I}$ are the coordinates defined in the material frame of reference. Throughout this work, lower case letters will refer to the spatial configuration, whereas upper case letters will refer to the material one.

Eq. 2a can be expressed as:

$$
p_{\mathrm{sl}}=\frac{1}{J}\left[\left(\lambda \ln (J)-\mu_{\mathrm{sl}}\right)+\frac{\mu_{\mathrm{sl}} b_{l l}}{n_{\mathrm{d}}}\right],
$$

and the deviatoric part, Eq. (2b), as:

$$
s_{\mathrm{sl}, i j}=\frac{\mu_{\mathrm{sl}}}{J}\left(b_{i j}-\frac{b_{l l}}{n_{\mathrm{d}}} \delta_{i j}\right) .
$$

Finally, by replacing Eq. (6) into our momentum equation we can write the resulting system of equations as an alternative to Eq. (1) as:

$$
\rho_{\mathrm{sl}} \partial_{t t} d_{i}-\partial_{j}\left[\frac{\mu_{\mathrm{sl}}}{J}\left(b_{i j}-\frac{b_{l l}}{n_{\mathrm{d}}} \delta_{i j}\right)\right]-\partial_{i} p_{\mathrm{sl}}=\rho_{\mathrm{sl}} f_{\mathrm{sl}, i}
$$




$$
\frac{J}{\lambda} p_{\mathrm{sl}}=\ln (J)+\frac{\mu_{\mathrm{sl}}}{\lambda}\left(\frac{b_{l l}}{n_{\mathrm{d}}}-1\right)
$$

where $b_{i j}$ is expressed in terms of the displacements $d_{i}$ and the density evolves as

$$
\rho_{\mathrm{sl}}=J \rho_{\mathrm{s} 1,0},
$$

where $\rho_{\mathrm{sl}, 0}$ is the density in the initial configuration. This is the two-field form of the solid mechanics equations we shall consider, the unknowns being $d_{i}$ and $p_{\mathrm{sl}}$. We shall write these unknowns as $\boldsymbol{y}_{\mathrm{sl}}=\left[\boldsymbol{d}, p_{\mathrm{sl}}\right]$, and denote by $\mathscr{A}\left(\boldsymbol{y}_{\mathrm{sl}}\right)$ the spatial nonlinear operator associated to Eqs. 77 , so that these equations can be written as

$$
\mathscr{M} \partial_{t t} \boldsymbol{y}_{\mathrm{sl}}+\mathscr{A}\left(\boldsymbol{y}_{\mathrm{sl}}\right)=\boldsymbol{F}_{\mathrm{sl}},
$$

where $\mathscr{M}:=\operatorname{diag}\left[\rho_{\mathrm{sl}} \boldsymbol{I}_{n_{\mathrm{d}}}, 0\right], \boldsymbol{I}_{n_{\mathrm{d}}}$ being the identity on vectors and $\boldsymbol{F}_{\mathrm{sl}}:=\left[\rho_{\mathrm{sl}} \boldsymbol{f}_{\mathrm{sl}}, 0\right]$.

\subsection{Linearization}

In order to approximate a solution to this highly non-linear problem we can re-write it by approximating our variables $\boldsymbol{y}_{\mathrm{sl}}=\left[\boldsymbol{d}, p_{\mathrm{sl}}\right]$ in terms of their increment $\delta \boldsymbol{y}_{\mathrm{sl}}=\left[\delta \boldsymbol{d}, \delta p_{\mathrm{sl}}\right]$ in the following form:

$$
\boldsymbol{y}_{\mathrm{sl}}=\tilde{\boldsymbol{y}}_{\mathrm{sl}}+\delta \boldsymbol{y}_{\mathrm{sl}},
$$

where $\tilde{\boldsymbol{y}}_{\mathrm{s} \mathrm{l}}=\left[\tilde{\boldsymbol{d}}, \tilde{p}_{\mathrm{sl}}\right]$ is the vector consisting of previously known values of $\boldsymbol{y}_{\mathrm{sl}}$. The Newton-Raphson linearization is obtained inserting this split in the equations to be solved and neglecting quadratic terms of the increments. Denoting with ${ }^{\sim}$ the functions computed with $\tilde{\boldsymbol{y}}_{\mathrm{sl}}$, Eqs. (7) can be re-written as (see e.g. [29] for the linearization of $J$ and of its logarithm):

$$
\begin{aligned}
\rho_{\mathrm{sl}} \partial_{t t} \delta d_{i} & -\partial_{j}\left[\frac{\mu_{\mathrm{sl}}}{\tilde{J}}\left(\frac{\partial \delta d_{i}}{\partial X_{K}} \tilde{F}_{j K}+\tilde{F}_{i K} \frac{\partial \delta d_{j}}{\partial X_{K}}\right)\right]+2 \frac{\delta_{i j}}{n_{\mathrm{d}}} \partial_{j}\left(\frac{\mu_{\mathrm{sl}}}{\tilde{J}} \tilde{F}_{l K} \frac{\partial \delta d_{l}}{\partial X_{K}}\right) \\
& +\partial_{j}\left[\frac{\mu_{\mathrm{sl}}}{\tilde{J}}\left(\tilde{F}_{K l}^{-1} \frac{\partial \delta d_{l}}{\partial X_{K}}\right)\left(\tilde{b}_{i j}-\frac{\tilde{b}_{l l}}{n_{\mathrm{d}}} \delta_{i j}\right)\right]-\partial_{i} \delta p_{\mathrm{sl}} \\
& =\rho_{\mathrm{sl}} f_{\mathrm{sl}, i}+\partial_{j}\left[\frac{\mu_{\mathrm{sl}}}{\tilde{J}}\left(\tilde{b}_{i j}-\frac{\tilde{b}_{l l}}{n_{\mathrm{d}}} \delta_{i j}\right)\right]+\partial_{i} \tilde{p}_{\mathrm{sl}}-\rho_{\mathrm{sl}} \partial_{t t} \tilde{d}_{i}, \\
\frac{\tilde{J}}{\lambda} \delta p_{\mathrm{sl}}+[ & \left.\left(\frac{\tilde{J} \tilde{p}_{\mathrm{sl}}}{\lambda}-1\right) \tilde{F}_{K l}^{-1}-\frac{2 \mu_{\mathrm{sl}}}{\lambda n_{\mathrm{d}}} \tilde{F}_{l K}\right] \frac{\partial \delta d_{l}}{\partial X_{K}}=-\frac{\tilde{J}}{\lambda} \tilde{p}_{\mathrm{sl}}+\ln (\tilde{J})+\frac{\mu_{\mathrm{sl}}}{\lambda}\left(\frac{\tilde{b}_{i i}}{n_{\mathrm{d}}}-1\right) .
\end{aligned}
$$

Let us denote by $\mathscr{L}_{\mathrm{sl}}\left(\tilde{\boldsymbol{y}}_{\mathrm{sl}} ; \delta \boldsymbol{y}_{\mathrm{sl}}\right)$ the spatial linear operator on $\delta \boldsymbol{y}_{\mathrm{sl}}$, for given $\tilde{\boldsymbol{y}}_{\mathrm{sl}}$, appearing in the left-hand-side (LHS) of these equations. Using the notation in Eq. [8], we may write

$$
\mathscr{M} \partial_{t t} \delta \boldsymbol{y}_{\mathrm{sl}}+\mathscr{L}_{\mathrm{sl}}\left(\tilde{\boldsymbol{y}}_{\mathrm{sl}} ; \boldsymbol{\delta} \boldsymbol{y}_{\mathrm{sl}}\right)=\boldsymbol{F}_{\mathrm{sl}}-\mathscr{M} \partial_{t t} \tilde{\boldsymbol{y}}_{\mathrm{sl}}-\mathscr{A}\left(\tilde{\boldsymbol{y}}_{\mathrm{sl}}\right) .
$$

In this way, upon convergence $\delta \boldsymbol{y}_{\mathrm{sl}} \approx \mathbf{0}$ and $\tilde{\boldsymbol{y}}_{\mathrm{sl}}$ is the solution of Eq. 8 .

\subsection{Initial and boundary-value problem}

The problem to be solved consists in finding $\left.\boldsymbol{y}_{\mathrm{sl}}: \Omega_{\mathrm{sl}} \times\right] 0, t_{\mathrm{f}}\left[\longrightarrow \mathbb{R}^{n_{\mathrm{d}}} \times \mathbb{R}\right.$ as the solution to Eq. 8) and such that

$$
\begin{array}{ll}
d_{i}=d_{i, D} & \text { on } \left.\Gamma_{\mathrm{sl}, D}, t \in\right] 0, t_{\mathrm{f}}[, \\
n_{\mathrm{sl}, j} \sigma_{i j}=t_{\mathrm{sl}, i} & \text { on } \left.\Gamma_{\mathrm{sl}, N}, t \in\right] 0, t_{\mathrm{f}}[, \\
d_{i}=d_{i}^{0} & \text { in } \Omega_{\mathrm{sl}}, t=0, \\
\dot{d}_{i}=\dot{d}_{i}^{0} & \text { in } \Omega_{\mathrm{sl}}, t=0,
\end{array}
$$


where $\boldsymbol{d}^{0}$ is a prescribed initial displacement, $\boldsymbol{d}^{0}$ is a prescribed initial velocity, $\boldsymbol{d}_{D}$ is a prescribed displacement on the boundary $\Gamma_{\mathrm{sl}, D}, \boldsymbol{t}_{\mathrm{sl}}$ is a prescribed traction on the boundary $\Gamma_{\mathrm{sl}, N}$, and $\boldsymbol{n}_{\mathrm{sl}}$ is the normal to the solid domain. Here it is assumed that $\Gamma_{\mathrm{sl}, D}$ and $\Gamma_{\mathrm{sl}, N}$ are a partition of $\partial \Omega_{\mathrm{sl}}$, although later we will also introduce the interface boundary with the fluid.

\subsection{Weak form}

Let $(\cdot, \cdot)_{\omega}$ the integral of the product of two functions, with the subscript omitted when $\omega=\Omega_{\mathrm{sl}}$. Let $Y_{\mathrm{sl}}$ be the space of functions where $\boldsymbol{y}_{\mathrm{sl}}$ must belong for each time $t$; functions in $Y_{\mathrm{sl}}$ satisfy the Dirichlet boundary conditions. Let also $Y_{\mathrm{sl}, 0}$ be the space of functions with the same regularity as $Y_{\mathrm{sl}}$ but satisfying the homogeneous counterpart of the Dirichlet boundary conditions.

The weak form of the two-field elasto-dynamic solid equations consists in finding $\left[\boldsymbol{d}, p_{\mathrm{sl}}\right]:\left[0, t_{\mathrm{f}}\right] \longrightarrow$ $Y_{\mathrm{sl}}$ such that

$$
\begin{aligned}
& \left(e_{i}, \rho_{\mathrm{sl}} \partial_{t t} \delta d_{i}\right)+\left(\frac{\partial e_{i}}{\partial x_{j}}, \frac{\mu_{\mathrm{sl}}}{\tilde{J}}\left(\frac{\partial \delta d_{i}}{\partial X_{K}} \tilde{F}_{j K}+\tilde{F}_{i K} \frac{\partial \delta d_{j}}{\partial X_{K}}\right)\right) \\
& -\left(\frac{\partial e_{i}}{\partial x_{i}}, 2 \frac{\mu_{\mathrm{sl}}}{\tilde{J} n_{\mathrm{d}}} \tilde{F}_{l K} \frac{\partial \delta d_{l}}{\partial X_{K}}\right)-\left(\frac{\partial e_{i}}{\partial x_{j}}, \frac{\mu_{\mathrm{sl}}}{\tilde{J}}\left(\tilde{F}_{K l}^{-1} \frac{\partial \delta d_{l}}{\partial X_{K}}\right)\left(\tilde{b}_{i j}-\frac{\tilde{b}_{l l}}{n_{\mathrm{d}}} \delta_{i j}\right)\right) \\
& +\left(\frac{\partial e_{i}}{\partial x_{i}}, \delta p_{\mathrm{sl}}\right)=\left(e_{i}, \rho_{\mathrm{sl}} f_{\mathrm{sl}, i}\right)+\left(e_{i}, t_{i}\right)_{\Gamma_{\mathrm{sl}, N}}-\left(\rho_{\mathrm{sl}} \partial_{t t} \tilde{d}_{i}, e_{i}\right) \\
& \quad-\left(\frac{\partial e_{i}}{\partial x_{j}}, \frac{\mu_{\mathrm{sl}}}{\tilde{J}}\left(\tilde{b}_{i j}-\frac{\tilde{b}_{l l}}{n_{\mathrm{d}}} \delta_{i j}\right)\right)-\left(\frac{\partial e_{i}}{\partial x_{i}}, \tilde{p}_{\mathrm{sl}}\right), \\
& \left(q_{\mathrm{sl}}, \frac{\tilde{J}}{\lambda} \delta p_{\mathrm{sl}}\right)+\left(q_{\mathrm{sl}},\left[\left(\frac{\tilde{J} \tilde{p}_{\mathrm{s}}}{\lambda}-1\right) \tilde{F}_{K l}^{-1}-\frac{2 \mu_{\mathrm{sl}}}{\lambda n_{\mathrm{d}}} \tilde{F}_{l K}\right] \frac{\partial \delta d_{l}}{\partial X_{K}}\right) \\
& =\left(q_{\mathrm{sl}},-\frac{\tilde{J}}{\lambda} \tilde{p}_{\mathrm{sl}}+\ln (\tilde{J})+\frac{\mu_{\mathrm{sl}}}{\lambda}\left(\frac{\tilde{b}_{i i}}{n_{\mathrm{d}}}-1\right)\right),
\end{aligned}
$$

for all $\left.\boldsymbol{z}_{\mathrm{sl}}=\left[\boldsymbol{e}, q_{\mathrm{sl}}\right] \in Y_{\mathrm{sl}, 0}, t \in\right] 0, t_{\mathrm{f}}[$, and satisfying initial conditions in a weak sense.

Let us define the form $B_{\mathrm{sl}}$ as:

$$
\begin{aligned}
& B_{\mathrm{sl}}\left(\tilde{\boldsymbol{y}}_{\mathrm{sl}} ; \delta \boldsymbol{y}_{\mathrm{sl}}, \boldsymbol{z}_{\mathrm{sl}}\right)=\left(\frac{\partial e_{i}}{\partial x_{j}}, \frac{\mu_{\mathrm{sl}}}{\tilde{J}}\left(\frac{\partial \delta d_{i}}{\partial X_{K}} \tilde{F}_{j K}+\tilde{F}_{i K} \frac{\partial \delta d_{j}}{\partial X_{K}}\right)\right)-\left(\frac{\partial e_{i}}{\partial x_{i}}, 2 \frac{\mu_{\mathrm{sl}}}{\tilde{J}_{n_{\mathrm{d}}}} \tilde{F}_{l K} \frac{\partial \delta d_{l}}{\partial X_{K}}\right) \\
& \quad-\left(\frac{\partial e_{i}}{\partial x_{j}}, \frac{\mu_{\mathrm{sl}}}{\tilde{J}}\left(\tilde{F}_{K l}^{-1} \frac{\partial \delta d_{l}}{\partial X_{K}}\right)\left(\tilde{b}_{i j}-\frac{\tilde{b}_{l l}}{n_{\mathrm{d}}} \delta_{i j}\right)\right)+\left(\frac{\partial e_{i}}{\partial x_{i}}, \delta p_{\mathrm{sl}}\right)+\left(q_{\mathrm{sl}}, \frac{\tilde{J}}{\lambda} \delta p_{\mathrm{sl}}\right) \\
& \quad+\left(q_{\mathrm{sl}},\left[\left(\frac{\tilde{J}_{\tilde{p}_{\mathrm{sl}}}}{\lambda}-1\right) \tilde{F}_{K l}^{-1}-\frac{2 \mu_{\mathrm{sl}}}{\lambda n_{\mathrm{d}}} \tilde{F}_{l K}\right] \frac{\partial \delta d_{l}}{\partial X_{K}}\right),
\end{aligned}
$$

and a form

$$
\begin{gathered}
L_{\mathrm{sl}}\left(\tilde{\boldsymbol{y}}_{\mathrm{sl}} ; \boldsymbol{z}_{\mathrm{sl}}\right)=\left(e_{i}, \rho_{\mathrm{sl}} f_{\mathrm{sl}, i}\right)+\left(e_{i}, t_{i}\right)_{\Gamma_{\mathrm{sl}, N}}-\left(\frac{\partial e_{i}}{\partial x_{j}}, \frac{\mu_{\mathrm{sl}}}{\tilde{J}}\left(\tilde{b}_{i j}-\frac{\tilde{b}_{l l}}{n_{\mathrm{d}}} \delta_{i j}\right)\right) \\
-\left(\frac{\partial e_{i}}{\partial x_{i}}, \tilde{p}_{\mathrm{sl}}\right)+\left(q_{\mathrm{sl}},-\frac{\tilde{J}}{\lambda} \tilde{p}_{\mathrm{sl}}+\ln (\tilde{J})+\frac{\mu_{\mathrm{sl}}}{\lambda}\left(\frac{\tilde{b}_{i i}}{n_{\mathrm{d}}}-1\right)\right),
\end{gathered}
$$

which enable us to write Eqs. 12 in the following simplified form:

$$
\left(\rho_{\mathrm{sl}} \partial_{t t} \delta d_{i}, e_{i}\right)+B_{\mathrm{sl}}\left(\tilde{\boldsymbol{y}}_{\mathrm{sl}} ; \delta \boldsymbol{y}_{\mathrm{sl}}, \boldsymbol{z}_{\mathrm{sl}}\right)=L_{\mathrm{sl}}\left(\tilde{\boldsymbol{y}}_{\mathrm{sl}} ; \boldsymbol{z}_{\mathrm{sl}}\right)-\left(\rho_{\mathrm{sl}} \partial_{t t} \tilde{d}_{i}, e_{i}\right),
$$

for all test functions $\boldsymbol{z}_{\mathrm{sl}}=\left[\boldsymbol{e}, q_{\mathrm{sl}}\right] \in Y_{\mathrm{sl}, 0}$. Initial conditions have to added to this variational equation. 


\subsection{Time discretization}

Let us consider a uniform partition of the time interval $] 0, t_{\mathrm{f}}[$ of size $\delta t$, and let us denote with superscript $n$ the time level. For the temporal discretization the following second order Backward Difference scheme (BDF2) has been used:

$$
\boldsymbol{a}^{n+1}=\frac{1}{\delta t^{2}}\left(2 \boldsymbol{d}^{n+1}-5 \boldsymbol{d}^{n}+4 \boldsymbol{d}^{n-1}-\boldsymbol{d}^{n-2}\right)
$$

where $\boldsymbol{d}^{n+1}$ and $\boldsymbol{a}^{n+1}$ are approximations to the position and acceleration $\left(\partial_{t t} \boldsymbol{d}\right)$ vectors at time $t^{n+1}=(n+1) \delta t$. Note that it is possible to use any other time integration scheme.

\subsection{Galerkin spatial discretization}

Let $\mathscr{P}_{h}$ denote a FE partition of the solid domain $\Omega_{\mathrm{sl}}$. The diameter of an element domain $K \in \mathscr{P}_{h}$ is denoted by $h_{K}$ and the diameter of the FE partition by $h=\max \left\{h_{K} \mid K \in \mathscr{P}_{h}\right\}$. We will define a subscript $h$ to refer to FE functions and spaces. We can construct the approximating space for the unknown and the test functions, $Y_{\mathrm{sl}, h}$ and $Y_{\mathrm{sl}, h, 0}$, respectively, in the usual manner. We consider here conforming approximations.

The Galerkin FE approximation to the problem can be written as: find $\boldsymbol{y}_{\mathrm{sl}, h}=\left[\boldsymbol{d}_{h}, p_{\mathrm{sl}, h}\right]:\left[0, t_{\mathrm{f}}\right] \longrightarrow$ $Y_{\mathrm{sl}, h}$ such that

$$
\left(\rho_{\mathrm{sl}} \partial_{t t} \delta \boldsymbol{d}_{h}, \boldsymbol{e}_{h}\right)+B_{\mathrm{sl}}\left(\tilde{\boldsymbol{y}}_{\mathrm{sl}, h} ; \delta \boldsymbol{y}_{\mathrm{sl}, h}, \boldsymbol{z}_{\mathrm{sl}, h}\right)=L_{\mathrm{sl}, h}\left(\tilde{\boldsymbol{y}}_{\mathrm{sl}} ; \boldsymbol{z}_{\mathrm{s} l, h}\right)-\left(\rho_{\mathrm{sl}} \partial_{t t} \tilde{\boldsymbol{d}}_{h}, \boldsymbol{e}_{h}\right),
$$

in $] 0, t_{\mathrm{f}}\left[\right.$ for all $\boldsymbol{z}_{\mathrm{sl}, h} \in Y_{\mathrm{sl}, h, 0}$, and satisfying the initial conditions weakly. Considering the problem discretized in time, at each time step the problem to be solved until convergence is

$$
\left(\rho_{\mathrm{sl}} \delta \boldsymbol{a}_{h}^{n+1}, \boldsymbol{e}_{h}\right)+B_{\mathrm{sl}}\left(\tilde{\boldsymbol{y}}_{\mathrm{sl}, h}^{n+1} ; \delta \boldsymbol{y}_{\mathrm{sl}, h}^{n+1}, \boldsymbol{z}_{\mathrm{sl}, h}\right)=L_{\mathrm{sl}, h}\left(\tilde{\boldsymbol{y}}_{\mathrm{sl}, h}^{n+1} ; \boldsymbol{z}_{\mathrm{sl}, h}\right)-\left(\rho_{\mathrm{sl}} \tilde{\boldsymbol{a}}_{h}^{n+1}, \boldsymbol{e}_{h}\right),
$$

for $n=1,2, \ldots$ and for all $\boldsymbol{z}_{\mathrm{sl}, h} \in Y_{\mathrm{sl}, h, 0}, \boldsymbol{d}_{h}^{0}$ and $\boldsymbol{d}_{h}^{1}$ being given by the initial conditions and $\boldsymbol{a}^{2}$ properly initialized (for example using a first order BDF scheme).

\subsection{A foreword on the VMS method}

Problem (17) is unstable, unless stringent requirements are met for the interpolating spaces of the variables in play (see, e.g., [25, 9]). In order to be able to use arbitrary interpolations, a stabilized FE method is required. The one we employ is based on the VMS concept, the key idea of which is to split the unknown of the problem as $\boldsymbol{y}=\boldsymbol{y}_{h}+\breve{\boldsymbol{y}}$, where $\boldsymbol{y}_{h}$ is the FE component and $\breve{\boldsymbol{y}}$ the sub-scale, i.e., the component that cannot be solved by the FE mesh and needs to be approximated.

We will not detail here how to obtain the final stabilized problem, which can be found in [29]. Let us just state this stabilized problem. Using orthogonal and dynamic sub-scales, this problem is:

$$
\begin{aligned}
\left(\rho \frac{\gamma_{1} \boldsymbol{y}_{h}}{\delta t^{2}}, \boldsymbol{z}_{h}\right)+B\left(\boldsymbol{y}_{h}, \boldsymbol{z}_{h}\right) & -\left(\Pi^{\perp}\left(\mathscr{L}\left(\boldsymbol{y}_{h}\right)\right), \boldsymbol{\tau}_{t} \mathscr{L}^{*}\left(\boldsymbol{z}_{h}\right)\right) \\
& =\left(\boldsymbol{f}+\rho \frac{\boldsymbol{y}_{h}^{\theta}}{\delta t^{2}}, \boldsymbol{z}_{h}\right)-\left(\rho \frac{\breve{\boldsymbol{y}}^{\theta}}{\delta t^{2}}, \boldsymbol{\tau}_{t} \mathscr{L}^{*}\left(\boldsymbol{z}_{h}\right)\right) \forall \boldsymbol{z}_{h},
\end{aligned}
$$

where $\mathscr{L}(\cdot)$ is now the linear operator of the problem being solved whereas $\mathscr{L}^{*}$ is its corresponding formal adjoint, $\mathscr{L}(\boldsymbol{y})=\boldsymbol{f}$ is the linear equation being approximated, $B$ the bilinear form defining the problem, $z_{h}$ the FE test function and $\Pi^{\perp}=I-\Pi_{h}, I$ being the identity operator and $\Pi_{h}$ the $L^{2}$ projection onto the FE space. If $\tau_{K}$ is the matrix of stabilization parameters (see Eqs. 22)- 23) below), $\boldsymbol{\tau}_{t}:=\left(\frac{\gamma_{1}}{\delta t^{2}} \boldsymbol{I}_{n_{\mathrm{d}}}+\boldsymbol{\tau}_{K}^{-1}\right)^{-1}, \boldsymbol{y}_{h}^{\theta}$ and $\breve{\boldsymbol{y}}^{\theta}$ are the collection of terms belonging to the previous time steps in our time integration scheme, for our FE scale and sub-scale respectively, and $\gamma_{1}$ is 
the coefficient multiplying the variable at step $n+1$. In this way, for a BDF2 scheme $\gamma_{1}=2$ and $\boldsymbol{y}^{\theta}=5 \boldsymbol{y}^{n}-4 \boldsymbol{y}^{n-1}+\boldsymbol{y}^{n-1}$ for $\boldsymbol{y}=\boldsymbol{y}_{h}$ or $\boldsymbol{y}=\breve{\boldsymbol{y}}$.

For the sake of clarity we assume that all terms are evaluated at time interval $n+1$, except the ones clearly specified, and $\Pi^{\perp}(\boldsymbol{f})=\mathbf{0}$, since the external forces either belong to the FE space or are approximated by elements in it, $\Pi^{\perp}\left(\rho \partial_{t t} \boldsymbol{y}_{h}\right)=\mathbf{0}$ since $\partial_{t t} \boldsymbol{y}_{h}$ is a FE function, and $\left(\rho \partial_{t t} \boldsymbol{y}_{h}, \breve{\boldsymbol{z}}\right)=0$ since these terms are mutually orthogonal. For more details on the orthogonal sub-scales see [8], and for the development of a stabilized general formulation for second order equations in time, see [29].

\subsection{Stabilization of the non-linear two-field solid problem}

Following the development from the previous section, we can express in particular the forms and operators for our stabilized two-field solid problem. If we consider the splitting of the unknown in the FE component and the sub-scale, instead of Eq. (17) we obtain:

$$
\begin{aligned}
& \left(\rho_{\mathrm{sl}} \delta \boldsymbol{a}_{h}, \boldsymbol{e}_{h}\right)+B_{\mathrm{sl}}\left(\tilde{\boldsymbol{y}}_{\mathrm{sl}} ; \delta \boldsymbol{y}_{\mathrm{sl}},\left[\boldsymbol{e}_{h}, 0\right]\right) \\
& \quad+\left(\frac{\partial e_{h, i}}{\partial x_{j}}, \frac{\mu_{\mathrm{sl}}}{\tilde{J}}\left(\frac{\partial \breve{d}_{i}}{\partial X_{K}} \tilde{F}_{j K}+\tilde{F}_{i K} \frac{\partial \breve{d}_{j}}{\partial X_{K}}\right)\right) \\
& \quad-\left(\frac{\partial e_{h, i}}{\partial x_{i}}, 2 \frac{\mu_{\mathrm{sl}}}{\tilde{J}_{n_{\mathrm{d}}}} \tilde{F}_{l K} \frac{\partial \breve{d}_{l}}{\partial X_{K}}\right)-\left(\frac{\partial e_{h, i}}{\partial x_{j}}, \frac{\mu_{\mathrm{sl}}}{\tilde{J}}\left(\tilde{F}_{K l}^{-1} \frac{\partial \breve{d}_{l}}{\partial X_{K}}\right)\left(\tilde{b}_{i j}-\frac{\tilde{b}_{l l}}{n_{\mathrm{d}}} \delta_{i j}\right)\right) \\
& \quad+\left(\frac{\partial e_{h, i}}{\partial x_{i}}, \breve{p}_{\mathrm{sl}}\right)=L_{\mathrm{sl}}\left(\tilde{\boldsymbol{y}}_{\mathrm{sl}} ;\left[\boldsymbol{e}_{h}, 0\right]\right)-\left(\rho_{\mathrm{sl}} \tilde{\boldsymbol{a}}_{h}, \boldsymbol{e}_{h}\right) \\
& B_{\mathrm{sl}}\left(\tilde{\boldsymbol{y}}_{\mathrm{sl}} ; \delta \boldsymbol{y}_{\mathrm{sl}},\left[\mathbf{0}, q_{\mathrm{sl} l, h}\right]\right)+\left(q_{h}, \frac{\tilde{J}}{\lambda} \breve{p}_{\mathrm{sl}}\right)+\left(q_{h},\left[\left(\frac{\tilde{J} \tilde{p}_{\mathrm{s} l, h}}{\lambda}-1\right) \tilde{F}_{K l}^{-1}-\frac{2 \mu_{\mathrm{sl}}}{\lambda n_{\mathrm{d}}} \tilde{F}_{l K}\right] \frac{\partial \breve{d}_{l}}{\partial X_{K}}\right) \\
& \quad=L_{\mathrm{sl}}\left(\tilde{\boldsymbol{y}}_{\mathrm{s} 1} ;\left[\mathbf{0}, q_{\mathrm{sl}, h}\right]\right),
\end{aligned}
$$

where the sub-scale at the current time step is $\breve{\boldsymbol{y}}_{\mathrm{sl}}=\left[\breve{\boldsymbol{d}}, \breve{p}_{\mathrm{sl}}\right]$. In these equations it is understood that all terms are evaluated at $t^{n+1}$, the variables with a tilde being guesses to the unknowns (from a previous iteration step, for example).

This version of the problem is unfeasible as it deals with derivatives of the sub-scales for which we do not have an approximation. These terms are integrated by parts and derivatives transferred to the test functions. The stabilization terms can then be written as:

$$
\sum_{K}\left(\breve{\boldsymbol{y}}_{\mathrm{s} 1, h}, \mathscr{L}_{\mathrm{sl}}^{*}\left(\tilde{\boldsymbol{y}}_{\mathrm{sl}} ; \boldsymbol{z}_{\mathrm{sl}, h}\right)\right)_{K}+\sum_{K}\left(\breve{\boldsymbol{y}}_{\mathrm{sl}}, \mathscr{F}_{\mathrm{sl}}^{*}\left(\tilde{\boldsymbol{y}}_{\mathrm{sl}} ; \boldsymbol{z}_{\mathrm{sl}, h}\right)\right)_{\partial K} \cdot
$$

We shall neglect the second term, although this approximation can be relaxed (see [11]) and, in fact, is needed if pressures are discontinuous. Concerning the first term, operator $\mathscr{L}_{\mathrm{sl}}^{*}\left(\tilde{\boldsymbol{y}}_{\mathrm{sl}} ; \boldsymbol{z}_{\mathrm{sl}, h}\right)$ has two components:

$$
\mathscr{L}_{\mathrm{sl}}^{*}\left(\tilde{\boldsymbol{y}}_{\mathrm{sl}} ; \boldsymbol{z}_{\mathrm{sl}, h}\right)=\left[\mathscr{L}_{\mathrm{sl}, 1}^{*}\left(\tilde{\boldsymbol{y}}_{\mathrm{sl}} ; \boldsymbol{z}_{\mathrm{sl}, h}\right), \mathscr{L}_{\mathrm{sl}, 2}^{*}\left(\tilde{\boldsymbol{y}}_{\mathrm{sl}} ; \boldsymbol{z}_{\mathrm{sl}, h}\right)\right]^{T},
$$

the first being a vector and the second a scalar, given by:

$$
\begin{aligned}
\mathscr{L}_{1}^{*}\left(\tilde{\boldsymbol{y}}_{\mathrm{sl}} ; \boldsymbol{z}_{\mathrm{s} 1, h}\right)= & -\frac{1}{2 \tilde{J}}\left[\frac{\partial}{\partial X_{K}}\left(\frac{\partial e_{h, i}}{\partial x_{j}} \tilde{F}_{j K}\right)+\frac{\partial}{\partial X_{K}}\left(\frac{\partial e_{h, j}}{\partial x_{i}} \tilde{F}_{j K}\right)\right]+\frac{1}{n_{\mathrm{d}} \tilde{J}} \frac{\partial}{\partial X_{K}}\left(\frac{\partial e_{h, l}}{\partial x_{l}} \tilde{F}_{i K}\right) \\
& -\frac{1}{2 \tilde{J}}\left(\frac{\partial}{\partial X_{K}}\left(\frac{\partial e_{h, l}}{\partial x_{j}}\left(\tilde{b}_{l j}-\frac{\tilde{b}_{n n}}{n_{\mathrm{d}}} \delta_{i j}\right) \tilde{F}_{K i}^{-1}\right)\right) \\
& -\frac{\partial}{\partial X_{K}}\left(\left(\frac{\tilde{J} \tilde{p}_{\mathrm{s} l, h}}{\lambda}-1\right) q_{h} \tilde{F}_{K i}^{-1}\right)+\left(\frac{2 \mu_{\mathrm{sl}}}{\lambda n_{\mathrm{d}}} \frac{\partial}{\partial X_{K}}\left(q_{h} \tilde{F}_{i K}\right)\right),
\end{aligned}
$$


$\mathscr{L}_{2}^{*}\left(\tilde{\boldsymbol{y}}_{\mathrm{sl}} ; \boldsymbol{z}_{\mathrm{sl}, h}\right)=\frac{\partial e_{h, i}}{\partial x_{i}}+\frac{\tilde{J}}{\lambda} q_{\mathrm{sl}}$.

To obtain the final expression of the stabilized problem, we need the expression of the sub-scales to be introduced in Eq. (20). For that, we just have to apply the general expressions obtained in the previous section, taking into account that we only have time derivatives of the displacements.

Using the same arguments as in [9], we will take the matrix of stabilization parameters within each element $K$ as

$$
\begin{aligned}
& \tau_{\mathrm{sl}, K}=\operatorname{diag}\left[\tau_{\mathrm{sl}, 1, K} \boldsymbol{I}_{n_{\mathrm{d}}}, \tau_{\mathrm{sl}, 2}\right], \\
& \tau_{\mathrm{sl}, 1, K}:=\left(c_{\mathrm{sl}, 1} \frac{\mu_{\mathrm{sl}}}{h_{K}^{2}}\right)^{-1}, \quad \tau_{\mathrm{sl}, 2}:=c_{\mathrm{sl}, 2} 2 \mu_{\mathrm{sl}},
\end{aligned}
$$

where $c_{\mathrm{sl}, 1}=4.0$ and $c_{\mathrm{sl}, 2}=0.1$ are numerical constants defined in the same way as in [6] for linear elements, $h_{K}$ being divided by the polynomial order for higher order interpolations.

The expression for the sub-scales is, finally:

$$
\begin{aligned}
& \breve{\boldsymbol{d}}^{n+1}=\tau_{\mathrm{sl}, 1, t}\left(\boldsymbol{R}_{\mathrm{sl}, 1}^{n+1}+\frac{\rho_{\mathrm{sl}} \breve{\boldsymbol{d}}^{\theta}}{\delta t^{2}}\right), \quad \tau_{\mathrm{sl}, 1, t}:=\left(\frac{\rho_{\mathrm{sl}} \gamma_{1}}{\delta t^{2}}+\tau_{\mathrm{sl}, 1, K}^{-1}\right)^{-1}, \\
& \breve{p}_{\mathrm{sl}}^{n+1}=\tau_{\mathrm{sl}, 2} R_{\mathrm{sl}, 2}^{n+1},
\end{aligned}
$$

where

$$
\boldsymbol{R}_{\mathrm{sl}}^{n+1}=\left[\boldsymbol{R}_{\mathrm{sl}, 1}^{n+1}, R_{\mathrm{sl}, 2}^{n+1}\right]^{T}=\boldsymbol{F}_{\mathrm{sl}}^{n+1}-\left[\rho_{\mathrm{sl}} \tilde{\boldsymbol{a}}_{\mathrm{sl}}^{n+1}, 0\right]^{T}-\mathscr{A}\left(\tilde{\boldsymbol{y}}_{\mathrm{sl}}^{n+1}\right)
$$

is the residual of the equation being solved at time step $n+1$, as it appears in Eq. (11).

The fully discrete and stabilized problem is now completely defined. It is given by equations (19), with the terms involving the subgrid scales given by the first term in Eq. (20), the adjoint operator given in Eqs. 21, the matrix of stabilization parameters in Eq. 22) and the sub-scales in Eqs. 24].

\section{FIELD TO FIELD FLUID STRUCTURE INTERACTION}

In this section we give a summary on our treatment on the different way to pair our FSI models, this being the standard model detailed in [30], the three-field FSI] shown in [29] and the novel two-field FSI introduced in this work. In what follows, we shall assume that there is a common moving boundary $\Gamma_{I}(t)$ between the solid and the fluid, so that the boundary of the solid domain $\Omega_{\mathrm{sl}}(t)$ is $\partial \Omega_{\mathrm{sl}}=\Gamma_{\mathrm{sl}, D} \cup \Gamma_{\mathrm{sl}, N} \cup \Gamma_{I}$, whereas the boundary of the fluid domain $\Omega_{\mathrm{ff}}(t)$ is $\partial \Omega_{\mathrm{fl}}=$ $\Gamma_{\mathrm{fl}, D} \cup \Gamma_{\mathrm{fl}, N} \cup \Gamma_{I}$, with void intersection between boundary components. Again, subscript $D$ refers to Dirichlet boundary conditions and subscript $N$ to Neumann boundary conditions, the same as described in previous sections.

\subsection{Flow problem}

The problem for the solid is given by the conservation equation (1), the mass conservation equation implied by the evolution of the solid density $\rho_{\mathrm{sl}}$ and the constitutive equation. As it has been explained, the alternatives to solve the problem are to use only the displacements as unknown (irreducible formulation), the displacements and the pressure (two-field formulation) and the displacements, the pressure and the deviatoric stress (three-field formulation). 
We will not detail the formulation for the fluid, which can be found in [29, 30], but we need to fix the notation. We consider an incompressible flow of a Newtonian fluid in a domain $\Omega_{\mathrm{ff}}$, the governing equations being written in terms of the velocity $\left.\boldsymbol{u}: \Omega_{\mathrm{ff}} \times\right] 0, t_{\mathrm{f}}\left[\longrightarrow \mathbb{R}^{n_{\mathrm{d}}}\right.$, the pressure $\left.p_{\mathrm{fl}}: \Omega_{\mathrm{fl}} \times\right] 0, t_{\mathrm{f}}\left[\longrightarrow \mathbb{R}\right.$ and, in the case of the three-field formulation, the deviatoric stress $s_{\mathrm{fl}}$ : $\left.\Omega_{\mathrm{fl}} \times\right] 0, t_{\mathrm{f}}\left[\longrightarrow \mathbb{R}^{n_{\mathrm{d}}} \otimes \mathbb{R}^{n_{\mathrm{d}}}\right.$. The field equations to be solved are:

$$
\begin{aligned}
\rho_{\mathrm{fl}} \partial_{t} \boldsymbol{u}+\rho_{\mathrm{fl}} \boldsymbol{u} \cdot \nabla \boldsymbol{u}-\nabla \cdot \boldsymbol{s}_{\mathrm{fl}}+\nabla p_{\mathrm{fl}} & =\rho_{\mathrm{fl}} \boldsymbol{f}, \\
\nabla \cdot \boldsymbol{u} & =0, \\
\frac{1}{2 \mu_{\mathrm{fl}}} \boldsymbol{s}_{\mathrm{fl}}-\nabla^{s} \boldsymbol{u} & =\mathbf{0},
\end{aligned}
$$

to be solved in $\left.\Omega_{\mathrm{f}} \times\right] 0, t_{\mathrm{f}}\left[\right.$, and with the expression of $s_{\mathrm{f}}$ in terms of $\boldsymbol{u}$ obtained from Eq. $25 \mathrm{c}$ inserted into Eq. 25a) to obtain the two-field formulation. In these equations, $\rho_{\mathrm{ff}}$ is the fluid density and $\mu_{\mathrm{fl}}$ the viscosity. As boundary and initial conditions we consider

$$
\begin{aligned}
\boldsymbol{u} & =\boldsymbol{u}_{D} & & \text { on } \left.\Gamma_{\mathrm{fl}, D}, t \in\right] 0, t_{\mathrm{f}}[, \\
\boldsymbol{n}_{\mathrm{fl}} \cdot \boldsymbol{\sigma}_{\mathrm{fl}} & =\boldsymbol{t}_{\mathrm{fl}} & & \text { on } \left.\Gamma_{\mathrm{fl}, N}, t \in\right] 0, t_{\mathrm{f}}[, \\
\boldsymbol{u} & =\boldsymbol{u}^{0} & & \text { in } \Omega_{\mathrm{fl}}, t=0,
\end{aligned}
$$

and the transmission conditions on $\Gamma_{I}$. The Cauchy stress tensor $\boldsymbol{\sigma}_{\mathrm{fl}}$ is given by $\boldsymbol{\sigma}_{\mathrm{fl}}=-p_{\mathrm{fl}} \boldsymbol{I}_{n_{\mathrm{d}}}+$ $2 \mu_{\mathrm{fl}} \nabla^{s} \boldsymbol{u}$ in the two-field formulation and by $\boldsymbol{\sigma}_{\mathrm{fl}}=-p_{\mathrm{fl}} \boldsymbol{I}_{n_{\mathrm{d}}}+\boldsymbol{s}_{\mathrm{ff}}$ in the three-field case. Note that the pressure in the fluid is minus the mean stress, whereas in the solid it is directly the mean stress. Here and below, $\boldsymbol{n}_{\mathrm{fl}}$ is the normal to the fluid domain.

Let $\boldsymbol{y}_{\mathrm{f}}^{(n)}$ be the unknown of the problem, where $\boldsymbol{y}_{\mathrm{f}}^{(2)}=\left[\boldsymbol{u}, p_{\mathrm{f}}\right]$ in the case of the two-field approach and $\boldsymbol{y}_{\mathrm{fl}}^{(3)}=\left[\boldsymbol{u}, \boldsymbol{s}_{\mathrm{f}}, p_{\mathrm{ff}}\right]$ in the three-field case. Let $Y_{\mathrm{fl}}^{(n)}$ be the spaces where these unknowns must belong to make well defined the variational problem written below, $n=2,3$, and let also $Y_{\mathrm{fl}, 0}^{(n)}$ be the space for the test functions $\boldsymbol{z}_{\mathrm{fl}}^{(n)}$, where $\boldsymbol{z}_{\mathrm{fl}}^{(2)}=\left[\boldsymbol{v}, q_{\mathrm{fl}}\right]$ and $\boldsymbol{z}_{\mathrm{fl}}^{(3)}=\left[\boldsymbol{v}, \boldsymbol{\xi}_{\mathrm{fl}}, q_{\mathrm{fl}}\right]$, $\boldsymbol{v}$ being the velocity test function, $\boldsymbol{\xi}_{\mathrm{fl}}$ the stress test function and $q_{\mathrm{fl}}$ the pressure test function. For a given velocity field $\hat{\boldsymbol{u}}$ we define the forms:

$$
\begin{aligned}
B_{\mathrm{f}}^{(2)}\left(\hat{\boldsymbol{u}} ; \boldsymbol{y}_{\mathrm{fl}}^{(2)}, \boldsymbol{z}_{\mathrm{f}}^{(2)}\right) & =\mu_{\mathrm{fl}}\left(\nabla^{s} \boldsymbol{v}, \nabla^{s} \boldsymbol{u}\right)+\left(\rho_{\mathrm{fl}} \hat{\boldsymbol{u}} \cdot \nabla \boldsymbol{u}, \boldsymbol{v}\right)-\left(p_{\mathrm{fl}}, \nabla \cdot \boldsymbol{v}\right)+\left(q_{\mathrm{fl}}, \nabla \cdot \boldsymbol{u}\right), \\
B_{\mathrm{fl}}^{(3)}\left(\hat{\boldsymbol{u}} ; \boldsymbol{y}_{\mathrm{fl}}^{(3)}, \boldsymbol{z}_{\mathrm{fl}}^{(3)}\right) & =\left(\boldsymbol{s}_{\mathrm{fl}}, \nabla^{s} \boldsymbol{u}\right)+\left(\rho_{\mathrm{fl}} \hat{\boldsymbol{u}} \cdot \nabla \boldsymbol{u}, \boldsymbol{v}\right)-\left(p_{\mathrm{fl}}, \nabla \cdot \boldsymbol{v}\right)+\left(q_{\mathrm{fl}}, \nabla \cdot \boldsymbol{u}\right) \\
& +\frac{1}{2 \mu_{\mathrm{fl}}}\left(\boldsymbol{s}_{\mathrm{fl}}, \boldsymbol{\xi}_{\mathrm{fl}}\right)-\left(\nabla^{s} \boldsymbol{u}, \boldsymbol{\xi}_{\mathrm{fl}}\right), \\
L_{\mathrm{fl}}\left(\boldsymbol{z}_{\mathrm{fl}}^{(n)}\right) & =\left(\rho_{\mathrm{fl}} \boldsymbol{f}, \boldsymbol{v}\right)+\left(\boldsymbol{t}_{\mathrm{fl}}, \boldsymbol{v}\right)_{\Gamma_{\mathrm{fl}, N}},
\end{aligned}
$$

where all integrals without subscript are understood to be performed on $\Omega_{\mathrm{sl}}$.

The two and three-field variational formulations of the incompressible Navier-Stokes can be written as follows: find $\left.\boldsymbol{y}_{\mathrm{fl}}^{(n)}:\right] 0, t_{\mathrm{fl}}\left[\longrightarrow Y_{\mathrm{fl}}^{(n)}\right.$ such that

$$
\left(\rho_{\mathrm{fl}} \partial_{t} \boldsymbol{u}, \boldsymbol{v}\right)+B_{\mathrm{fl}}^{(n)}\left(\boldsymbol{u} ; \boldsymbol{y}_{\mathrm{fl}}^{(n)}, \boldsymbol{z}_{\mathrm{fl}}^{(n)}\right)=L_{\mathrm{fl}}\left(\boldsymbol{z}_{\mathrm{fl}}^{(n)}\right) \quad \forall \boldsymbol{z}_{\mathrm{fl}}^{(n)} \in Y_{\mathrm{fl}, 0}^{(n)},
$$

with $n=2$ or 3 , and satisfying the initial conditions in a weak sense.

The FE approximation we use for problem (27) can be found in [29, 30].

\subsection{Solid problem}

The problem for the solid has been set in Section 2, where the two-field approach has been detailed. Now we wish to consider the one-field (displacements), two-field (displacements, pressure) and three-field (displacement, deviatoric stresses, pressure) approaches. Let $\boldsymbol{y}_{\mathrm{sl}}^{(n)}$ the unknown in 
each case, where $\boldsymbol{y}_{\mathrm{sl}}^{(1)}=\boldsymbol{d}, \boldsymbol{y}_{\mathrm{sl}}^{(2)}=\left[\boldsymbol{d}, p_{\mathrm{sl}}\right]$ and $\boldsymbol{y}_{\mathrm{sl}}^{(3)}=\left[\boldsymbol{d}, \boldsymbol{s}_{\mathrm{sl}}, p_{\mathrm{sl}}\right]$. The stress components are given in Eq. 6. 6et $Y_{\mathrm{sl}}^{(n)}$ be the spaces where $\boldsymbol{y}_{\mathrm{sl}}^{(n)}$ must belong to have a well defined variational problem, $n=1,2,3, \boldsymbol{z}_{\mathrm{sl}}^{(n)}$ the corresponding test functions, with $\boldsymbol{z}_{\mathrm{sl}}^{(1)}=\boldsymbol{e}, \boldsymbol{z}_{\mathrm{sl}}^{(2)}=\left[\boldsymbol{e}, q_{\mathrm{sl}}\right]$ and $\boldsymbol{z}_{\mathrm{sl}}^{(3)}=$ $\left[\boldsymbol{e}, \boldsymbol{\xi}_{\mathrm{sl}}, q_{\mathrm{sl}}\right]$, and $Y_{\mathrm{sl}, 0}^{(n)}$ the spaces for the test functions, $n=1,2,3$.

The variational formulation for the solid, written in incremental (linearized) form, can be written as follows: find $\left.\boldsymbol{y}_{\mathrm{sl}}^{(n)}:\right] 0, t_{\mathrm{fl}}\left[\longrightarrow Y_{\mathrm{sl}}^{(n)}\right.$ as the converged solution $\left(\delta \boldsymbol{y}_{\mathrm{sl}}^{(n)}=\mathbf{0}\right)$ to the problem

$$
\left(\rho_{\mathrm{sl}} \partial_{t t} \delta \boldsymbol{d}, \boldsymbol{e}\right)+B_{\mathrm{sl}}^{(n)}\left(\tilde{\boldsymbol{y}}_{\mathrm{sl}} ; \delta \boldsymbol{y}_{\mathrm{sl}}^{(n)}, \boldsymbol{z}_{\mathrm{sl}}^{(n)}\right)=L_{\mathrm{sl}}\left(\boldsymbol{z}_{\mathrm{sl}}^{(n)}\right)-\left(\rho_{\mathrm{fl}} \partial_{t t} \tilde{\boldsymbol{d}}, \boldsymbol{e}\right) \quad \forall \boldsymbol{z}_{\mathrm{sl}}^{(n)} \in Y_{\mathrm{sl}, 0}^{(n)},
$$

with $n=1,2$ or 3, and satisfying the initial conditions in a weak sense. All integrals involved in this equation are assumed to be performed on $\Omega_{\mathrm{sl}}$, except that done on $\Gamma_{\mathrm{sl}, N}$. The notation involved in this equation is the same as in Section 2. In particular, Eq. (15) corresponds exactly to Eq. (28) in the case $n=2$.

The forms $B_{\mathrm{sl}}^{(n)}\left(\tilde{\boldsymbol{y}}_{\mathrm{sl}} ; \delta \boldsymbol{y}_{\mathrm{sl}}^{(n)}, \boldsymbol{z}_{\mathrm{sl}}^{(n)}\right)$ in the cases $n=1$ and $n=3$ are easily obtained either expressing the pressure in terms of the displacements for $n=1$ or introducing the deviatoric stresses as a variable in the case $n=3$. Details and the FE approximation can be found in [30] for $n=1$ and in [29] for $n=3$.

\subsection{Weak form of the Field to Field coupled problem}

As an interaction problem, each one of the individual problems is treated naturally in different frames of reference. While the mesh tracks the solid particles, it does not track the fluid. The approach followed in this work can be taken as the traditional in a broad sense, where the fluid is treated by means of an Arbitrary-Lagrangian-Eulerian (ALE) formulation while the solid follows the usual Updated Lagrangian approach. [13] explains in a detailed manner the ALE formulation, as well as its advantages and disadvantages. The mesh movement algorithm has been taken from [7], which has proven simple, robust and reliable. In what follows $c$ denotes the convection velocity from the mesh point of view, which is given by $\boldsymbol{c}=\boldsymbol{u}-\boldsymbol{u}_{\text {mesh }}$, where $\boldsymbol{u}_{\text {mesh }}$ is the velocity of the points in the computational domain to which the unknowns are referred. Also note that in this form, the domains to which the fluid and solid pertain, $\Omega_{\mathrm{fl}}(t)$ and $\Omega_{\mathrm{sl}}(t)$, respectively, are now time dependent, as they change according to what the ALE formulation dictates in the fluid and the updated Lagrangian approach yields in the solid.

In order to ensure accurate and stable dynamic simulations of [FSI problems, dynamic and kinematic continuity should be guaranteed on the interaction interface for partitioned problems. Monolithic schemes naturally satisfy these conditions. Kinematic and dynamic continuity require that displacements and tractions be continuous at the interface, respectively, energy conservation requires that both conditions be satisfied simultaneously [32]. These conditions read:

$$
\begin{array}{ll}
\boldsymbol{u}=\partial_{t} \boldsymbol{d} & \text { on } \Gamma(t)_{\mathrm{I}}, \\
\boldsymbol{n}_{\mathrm{sl}} \cdot \boldsymbol{\sigma}_{\mathrm{sl}}+\boldsymbol{n}_{\mathrm{fl}} \cdot \boldsymbol{\sigma}_{\mathrm{fl}}=\mathbf{0} & \text { on } \Gamma(t)_{\mathrm{I}}
\end{array}
$$

Since we will use an iteration-by-subdomain strategy, with Dirichlet conditions for the fluid (given by the displacement computed from the solid) and Neumann conditions for the solid (given by the stresses computed from the fluid), $\Gamma_{I}$ will be part of $\Gamma_{\mathrm{fl}, D}$ when computing the fluid and part of $\Gamma_{\mathrm{sl}, N}$ when computing the solid. This means that $Y_{\mathrm{fl}}^{(n)}$ and $Y_{\mathrm{fl}, 0}^{(n)}$ need to be properly modified to account for the Dirichlet conditions on $\Gamma_{I}$.

Let us write the variational form of the FSI problem to be solved taking into account the previous considerations. We state the continuous problem, the FE approximation being done according to what has been explained in Section 2 and in [29, 30]. This general problem can be stated as follows: find $\left.\left[\boldsymbol{y}_{\mathrm{fl}}^{(n)}, \boldsymbol{y}_{\mathrm{sl}}^{(m)}\right]:\right] 0, t_{\mathrm{f}}\left[\longrightarrow Y_{\mathrm{fl}}^{(n)} \times Y_{\mathrm{sl}}^{(m)}\right.$ such that 
Fluid equations:

$$
\begin{aligned}
& \boldsymbol{u}=\partial_{t} \boldsymbol{d} \quad \text { on } \Gamma_{I}, \\
& \left(\rho_{\mathrm{f}} \partial_{t} \boldsymbol{u}, \boldsymbol{v}\right)+B_{\mathrm{fl}}^{(n)}\left(\boldsymbol{u} ; \boldsymbol{y}_{\mathrm{fl}}^{(n)}, \boldsymbol{z}_{\mathrm{fl}}^{(n)}\right)=L_{\mathrm{fl}}\left(\boldsymbol{z}_{\mathrm{fl}}^{(n)}\right), \\
& \text { for all } \boldsymbol{z}_{\mathrm{fl}}^{(n)} \in Y_{\mathrm{fl}, 0}^{(n)}, \text { with } \boldsymbol{v}=\mathbf{0} \text { on } \Gamma_{\mathrm{fl}, D} \cup \Gamma_{I} .
\end{aligned}
$$

Solid equations:

$$
\left(\rho_{\mathrm{sl}} \partial_{t t} \delta \boldsymbol{d}, \boldsymbol{e}\right)+B_{\mathrm{sl}}^{(m)}\left(\tilde{\boldsymbol{y}}_{\mathrm{sl}} ; \delta \boldsymbol{y}_{\mathrm{sl}}^{(m)}, \boldsymbol{z}_{\mathrm{sl}}^{(m)}\right)=L_{\mathrm{sl}}\left(\boldsymbol{z}_{\mathrm{sl}}^{(m)}\right)-\left(\rho_{\mathrm{fl}} \partial_{t t} \tilde{\boldsymbol{d}}, \boldsymbol{e}\right)-\left(\boldsymbol{e}, \boldsymbol{n}_{\mathrm{fl}} \cdot \boldsymbol{\sigma}_{\mathrm{fl}}\right)_{\Gamma_{I}},
$$

for all $\boldsymbol{z}_{\mathrm{sl}}^{(m)} \in Y_{\mathrm{sl}, 0}^{(m)}$.

The case $n=2, m=1$ corresponds to the standard FSI problem. In this paper we aim to explore the behavior of the F2F cases $n=m=2$ and $n=m=3$.

Although we will not detail the FE implementation of problem (31), let us comment on a particularly relevant aspect of the $\overline{F 2 F}$ approach in FSI compared to the standard coupling, which is the implementation of transmission conditions. Suppose first that the meshes for the solid and the fluid match at $\Gamma_{I}$, and that stresses from the fluid need to be transferred to the solid as is usual. Suppose also that a nodal Lagrangian interpolation is used. In a classical velocity-pressure approach (standard FSI], normal stresses at the numerical integration points have to be transmitted; these are computed from pressures and velocity gradients interpolated from the nodes to the integration points. In a three-field approach, stresses are directly available at the nodes. If the meshes for the solid and the fluid do not match at $\Gamma_{I}$, there is an additional interpolation step. In a velocity-pressure approach, normal stresses at the integration points of the fluid have to transferred to the nodes of the solid, and from these to the integration points of the solid. In a three-field approach, normal stresses at the nodes of the fluid have to be transferred to the nodes of the solid; the number of operations involved in this case is significantly smaller than in the former. The two-field approach for the solid is obviously in between with the availability of the pressure at the nodes but the necessity to interpolate displacement gradients from the Gauss points to reconstruct the stress tensor. In every case, the transfer of information can be used using the standard Lagrangian interpolation, as done in our implementation, or by imposing restrictions, as explained in [14].

\subsection{Block iterative scheme}

Once Eqs. (31) have been discretized in time and space, they can be solved in a monolithic way, i.e., solving for $\left[\boldsymbol{y}_{\mathrm{fl}}^{(n)}, \boldsymbol{y}_{\mathrm{sl}}^{(m)}\right]$ at the same time, or in a segregated way, solving first for $\boldsymbol{y}_{\mathrm{fl}}^{(n)}$ and then for $\boldsymbol{y}_{\mathrm{sl}}^{(m)}$. This last possibility has in turn two options, a staggered one, in which at a given time step the time discrete version of the nonlinear problem (31a)- (31b) is solved with $\boldsymbol{d}$ computed from previous time steps and then Eq. (31c) is solved, and a fully coupled one, in which block iterations are performed to achieve full coupling of the equations at each time step. This last option is the one adopted in this work, known to be more stable than the staggered version.

Let $\delta_{t}$ be finite a difference approximations to $\partial_{t}$ and $\boldsymbol{a}$ an approximation for the solid acceleration at a certain time step $s$. To lighten the notation, no reference will be made to this time step, being understood that all variables are evaluated there. If superscript $k$ denotes the block-iterative counter, the problem we solve at each time step is: from known $\left[\boldsymbol{y}_{\mathrm{fl}}^{(n), k-1}, \boldsymbol{y}_{\mathrm{sl}}^{(m), k-1}\right] \in Y_{\mathrm{fl}}^{(n)} \times Y_{\mathrm{sl}}^{(m)}$, find $\left[\boldsymbol{y}_{\mathrm{fl}}^{(n), k}, \boldsymbol{y}_{\mathrm{sl}}^{(m), k}\right] \in Y_{\mathrm{fl}}^{(n)} \times Y_{\mathrm{sl}}^{(m)}$ such that

$$
\begin{aligned}
& \boldsymbol{u}^{k}=\delta_{t} \boldsymbol{d}^{k-1} \quad \text { on } \Gamma_{I}, \\
& \left(\rho_{\mathrm{fl}} \delta_{t} \boldsymbol{u}^{k}, \boldsymbol{v}\right)+B_{\mathrm{fl}}^{(n)}\left(\boldsymbol{u}^{k} ; \boldsymbol{y}_{\mathrm{fl}}^{(n), k}, \boldsymbol{z}_{\mathrm{fl}}^{(n)}\right)=L_{\mathrm{fl}}\left(\boldsymbol{z}_{\mathrm{fl}}^{(n)}\right), \\
& \text { for all } \boldsymbol{z}_{\mathrm{fl}}^{(n)} \in Y_{\mathrm{fl}, 0}^{(n)}, \text { with } \boldsymbol{v}=\mathbf{0} \text { on } \Gamma_{\mathrm{fl}, D} \cup \Gamma_{I} . \\
& \left(\rho_{\mathrm{sl}} \delta \boldsymbol{a}^{k}, \boldsymbol{e}\right)+B_{\mathrm{sl}}^{(m)}\left(\tilde{\boldsymbol{y}}_{\mathrm{sl}}^{k} ; \delta \boldsymbol{y}_{\mathrm{sl}}^{(m), k}, \boldsymbol{z}_{\mathrm{sl}}^{(m)}\right)=L_{\mathrm{sl}}\left(\boldsymbol{z}_{\mathrm{sl}}^{(m)}\right)-\left(\rho_{\mathrm{fl}} \tilde{\boldsymbol{a}}^{k}, \boldsymbol{e}\right)-\left(\boldsymbol{e}, \boldsymbol{n}_{\mathrm{fl}} \cdot \boldsymbol{\sigma}_{\mathrm{fl}}^{k}\right)_{\Gamma_{I}},
\end{aligned}
$$




$$
\text { for all } \boldsymbol{z}_{\mathrm{sl}}^{(m)} \in Y_{\mathrm{sl}, 0}^{(m)}
$$

where the stresses $\boldsymbol{\sigma}_{\mathrm{f}}^{k}$ are computed with the unknown computed in Eqs. $32 \mathrm{a}-\left(32 \mathrm{~b}, \boldsymbol{y}_{\mathrm{f}}^{(n), k}\right.$. Note that both problems (32b) and (32c) are non-linear, thus requiring inner iterations each, unless the linearization of the individual problems is coupled with the block-iterative scheme.

After each iteration of (32), we use a relaxation scheme that depends on a relaxation parameter. This can either be chosen from careful study of the problem at hand (a tiresome method an often inefficient) or can be calculated through optimal algorithms that calculate it automatically without any user input. In this respect, we have used a relaxation of the position and velocity of the interface boundary that the solid solver transmits to the fluid solver. We denote this position as $\boldsymbol{d}_{\Gamma_{\mathrm{I}}}$; from it, one may compute the velocity of the fluid boundary and $\boldsymbol{u}_{\text {mesh }}$, as explained above. We have implemented an Aitken relaxation scheme, in particular Aitken $\Delta^{2}$, detailed in [19], which is a reformulation from the algorithm initially proposed in [17]. Suppose that from values at the $(k-1)$ th iteration, the solid is solved, obtaining the boundary displacements $d_{\Gamma_{\mathrm{I}}, \mathrm{S}}^{k}$. Then, the fluid is solved from the boundary displacements $\boldsymbol{d}_{\Gamma_{\mathrm{I}}}^{k}$ computed as

$$
\boldsymbol{d}_{\Gamma_{\mathrm{I}}}^{k}=\boldsymbol{d}_{\Gamma_{\mathrm{I}}}^{k-1}+\omega^{k} \boldsymbol{r}_{\Gamma_{\mathrm{I}}}^{k}
$$

where

$$
\begin{aligned}
& \boldsymbol{r}_{\Gamma_{\mathrm{I}}}^{k}:=\boldsymbol{d}_{\Gamma_{\mathrm{I}}, \mathrm{s}}^{k}-\boldsymbol{d}_{\Gamma_{\mathrm{I}}}^{k-1}, \\
& \omega^{k}=-\omega^{k-1} \frac{\left(\boldsymbol{r}_{\Gamma_{\mathrm{I}}}^{k-1}\right)^{T}\left(\boldsymbol{r}_{\Gamma_{\mathrm{I}}}^{k}-\boldsymbol{r}_{\Gamma_{\mathrm{I}}}^{k-1}\right)}{\left|\boldsymbol{r}_{\Gamma_{\mathrm{I}}}^{k}-\boldsymbol{r}_{\Gamma_{\mathrm{I}}}^{k-1}\right|^{2}} .
\end{aligned}
$$

The general[FSI algorithm is essentially the same as Algorithm 1 shown in [30].

\section{REDUCED ORDER MODELING IN FSI}

Strongly coupled partitioned $\mathrm{FSI}$ algorithms may require a high number of iterations and relaxation, making the problem potentially expensive numerically and requiring long solution times. Previously we had introduced ROM into standard FSI] successfully [30], however later we noticed that the interaction between the fluid and solid domains can benefit from a mixed formulation approach [29]. It is our goal to examine how, if possible, our VMS ROM approach benefits from our previously developed F2F pairing. In this section we give a short review of the methodology we apply and the algorithmic aspects that concern it.

\subsection{Some ROM theory and notation}

Let us consider a high dimensional space $Y_{h}$ of dimension $R$, with $\varphi=\left\{\varphi^{1}, \ldots, \varphi^{R}\right\}$ its orthonormal basis, whose elements are vectors of $D$ components. We think of $Y_{h}$ as the FE space from which we construct our FOM, and thus $\varphi$ can be thought as an orthonormal basis obtained from the standard FE basis functions. Then any element $\boldsymbol{y}_{h} \in Y_{h}$ can be written as the linear combination

$$
\boldsymbol{y}_{h}=\sum_{k=1}^{R}\left(\boldsymbol{y}_{h}, \varphi^{k}\right) \boldsymbol{\varphi}^{k},
$$

now with $(\cdot, \cdot)$ the $L^{2}$-inner product in $Y_{h}$, perhaps weighted by a symmetric and positivedefinite matrix. We can also define a low-dimensional subspace $Y_{\text {rom }} \subset Y_{h}$ of dimension $r$, which approximates $Y_{h}$ as $r \rightarrow R$, with a basis $\phi=\left\{\phi^{1}, \ldots, \phi^{r}\right\}$. Using this basis, we can approximate 
any element $\boldsymbol{y}_{h}$ as

$$
\boldsymbol{y}_{h} \approx \boldsymbol{y}_{\mathrm{rom}}=\sum_{k=1}^{r} \phi^{k} a^{k},
$$

where $a^{k}$ is the $k$-th coefficient which can be computed as $a^{k}=\left(\boldsymbol{y}_{h}, \boldsymbol{\phi}^{k}\right)$, and which will typically be obtained from the solution of the reduced problem when $\boldsymbol{y}_{h}$ is unknown. The accuracy of the approximation depends on how well the basis $\phi$ approximates the exact basis $\varphi$; as we use a nested basis scheme, a finer basis includes the previous one within its space.

\subsection{Construction of the basis}

The method we use to construct the basis of the low-dimensional space is the Proper Orthogonal Decomposition (POD). The objective of this method is finding a basis from a collection of highfidelity "snapshots", which in our context are solutions in $Y_{h}$ of an evolution problem at certain time steps. As it is well known, we need to substract the mean. Thus, taking a set of data as a collection of $N$ snapshots $\left\{\boldsymbol{s}_{j}\right\}_{j=1}^{N}=\left\{\boldsymbol{y}_{h, j}-\overline{\boldsymbol{y}}_{h}\right\}_{j=1}^{N}$, the overbar denoting the mean of the $\boldsymbol{y}_{h, j} \in Y_{h}$, we can reproduce any element of this collection as

$$
\boldsymbol{y}_{h, j} \approx \overline{\boldsymbol{y}}_{h}+\sum_{k=1}^{r}\left(\boldsymbol{s}_{j}, \boldsymbol{\phi}^{k}\right) \boldsymbol{\phi}^{k}
$$

where, in the case of POD $\left\{\phi^{k}\right\}_{k=1}^{r}$ is an orthonormal system of $Y_{h}$ obtained from finding the orthonormal basis $\left\{\phi^{k}\right\}_{k=1}^{r}$ of $Y_{\text {rom }}$ such that:

$$
\begin{aligned}
& \min _{\{\phi\}_{k=1}^{r}} \frac{1}{N} \sum_{j=1}^{N}\left\|\boldsymbol{s}_{j}-\sum_{k=1}^{r}\left(\boldsymbol{s}_{j}, \boldsymbol{\phi}^{k}\right) \boldsymbol{\phi}^{k}\right\|^{2}, \\
& \text { subject to }\left(\boldsymbol{\phi}^{i}, \boldsymbol{\phi}^{j}\right)=\delta_{i j}, \quad 1 \leq i, j \leq r,
\end{aligned}
$$

where $\|\cdot\|$ denotes the $L^{2}$ norm. By means of a Singular Value Decomposition (SVD) we can solve for the basis $\left\{\phi^{k}\right\}_{k=1}^{r}$ from the matrix of snapshots. This basis depends on parameters as time-step, how often the snapshots were acquired and the reproducibility of the function being analyzed. A reduced basis can be defined by truncating the left singular-vectors at the $r$-th column. As a criterion for the truncation, we use the retained energy $\eta$, defined in [27] as:

$$
\eta=\left(\sum_{k=1}^{r} \lambda^{k}\right)\left(\sum_{k=1}^{R} \lambda^{k}\right)^{-1}
$$

where $\left\{\lambda^{k}\right\}_{k=1}^{R}$ are the singular values of the SVD The SVD produces a diagonal matrix which contains, from greatest to smallest, the eigenvalues of the associated (singular) eigen-vectors. The ordering of the eigenvalues is a measure of the relative importance of each of the basis functions in the whole system. In general, in a reducible problem (a problem that should be easily reproduced by means of ROM they decrease quickly in magnitude. If $r$ is sufficiently small, the time to compute the reduced system is minimal. The stage of the problem in which the basis is calculated is termed as the off-line phase.

In our context, $Y_{h}$ may be either the FE space for the solid or for the fluid, and $\boldsymbol{y}_{h}$ may be either $\boldsymbol{y}_{\mathrm{fl}, h}^{(n)}$ or $\boldsymbol{y}_{\mathrm{sl}, h}^{(m)}$, i.e., the FE approximations to $\boldsymbol{y}_{\mathrm{fl}}^{(n)}$ and $\boldsymbol{y}_{\mathrm{sl}}^{(m)}$ introduced in the last section. We compute independently the ROM space for each of these two problems. For details on how we calculate the basis for each domain, see [30]. 


\subsection{VMS-ROM}

In FSI problems and with the partitioned strategy we have followed, we have to solve one variational problem in the fluid domain and another one in the solid domain. The Galerkin FE approximation to these problems can be stated as these variational problems restricted to the FE spaces, both for the unknowns and for the test functions. In a similar way, the ROM could be expressed as the same variational problems, now restricting unknowns and test functions to the ROM spaces, with a much smaller dimension than the FE spaces. There are other options to state the ROM problem, but the approach described justifies that one may expect similar instability problems for the Galerkin FE method and the described Galerkin-ROM. Therefore, some sort of numerical stabilization will be required for the fluid problem and the mixed formulation for the solid.

The approach we shall follow for the fluid is the same as for the FE problem, namely, to use a VMS method with an approximation to the sub-grid scales similar to that given by Eq. (18). In the ROM case, it is particularly natural to use orthogonal sub-grid scales, since the vectors of the basis are mutually orthogonal. Therefore, if the ROM space is obtained by truncating the vectors obtained from a SVD of the collection of snapshots to the first $r$ members, the space of sub-grid scales is simply its $L^{2}$-orthogonal complement. See [23, 22] for an elaboration of this idea, that in this work we apply to both the solid and the fluid problem.

At the reduced level it is necessary to still assemble the linear system of unknowns to preserve the efficiency and accuracy of the method; this is a costly procedure as for nonlinear problmes it involves the whole array of unknowns and in general it is not scalable. Therefore it is generally critical to think about hyper-reduction as a means to placate this issue. A classical way to do this is by means of a selection of critical points that represent the main part of our domain and evaluate the nonlinear terms at those points. Such an approach can be termed hyper-reduction and there are various available techniques, like Gappy-POD [4], APHR [26] or GNAT [5]; an alternative is the recently proposed hyper-reduction by means of adaptive mesh refinement [22]. However our goal is of preliminary investigation on our F2F|FSI ROM scheme, and no hyper-reduction has been used in the numerical examples presented in Section 5.

\subsection{A word on error measurement}

Let us conclude this section indicating how we measure errors in Section 5. We apply the Root Mean Square Deviation (RMSD) to compare results obtained with the ROM to those obtained with the FOM. This way of measuring error is helpful because it shows 'how far' the solution is from our full order solution. In this way results presented here are not to be analyzed as a convergence of the method but rather as how well the reduced results reproduce the desired full order problem. The expression for the RMSD is given as:

$$
R M S D=\sqrt{\frac{1}{S} \sum_{j=1}^{S}\left|y_{R O M}^{j}-y_{F O M}^{j}\right|^{2}},
$$

where $y_{R O M}^{j}$ and $y_{F O M}^{j}$ are the variable that we want to compare in $\mathrm{ROM}$ and $\mathrm{FOM}$, respectively, either in the fluid or in the solid, evaluated at time step $j, S$ being the total number of time steps used for comparison.

\section{NUMERICAL RESULTS}

The first numerical example we present is a simple benchmark for the new solid two-field formulation introduced in this paper, which we compare with the one-field and three-field 
approaches; both the FOM and the ROM are considered. The next example is a FSI problem in which we compare the couplings discussed in this paper, namely, the standard one ( $n=2, m=1$ with the terminology of Section 3) and the F2F coupling ( $n=m=2$ and $n=m=3$ ); again, both the FOM and the ROM are considered. Finally, we extend the discussion to a 3D test.

\subsection{A simple test: dynamic oscillation of a bar}

This test is a rather simple one in which the purpose is to assess the behavior of all three solid formulations (one, two and three-field approaches), comparing them first in the full order space and then in the reduced order space. The test consists of a cantilevered bar subject to the force of gravity, being initially undeformed at rest. Only a short interval of the oscillation will be analyzed. The geometry and initial position of the bar are shown in Fig. 1] the physical parameters of the bar are given in Table I. where $\nu_{\mathrm{sl}}$ is Poisson's coefficient, and the legend for the results is given in Table

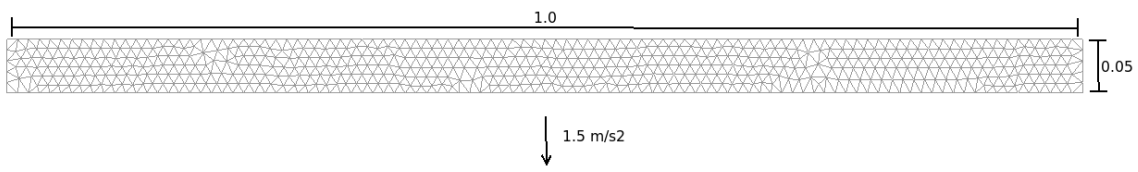

Figure 1. Geometry

Table I. Physical parameters

\begin{tabular}{cc}
\hline$\rho_{\mathrm{sl}}$ & $1000.0\left[\mathrm{Kg} / \mathrm{m}^{3}\right]$ \\
$\mu_{\mathrm{sl}}$ & $1.9310^{6}[\mathrm{~Pa}]$ \\
$\nu_{\mathrm{sl}}$ & 0.4 \\
Model & Neo-Hookean \\
\hline
\end{tabular}

Table II. Case parameters

\begin{tabular}{ccc|c}
\hline Name & Formulation & Space & Energy(\%) \\
\hline irr_fom & Irreducible & $\overline{\text { FOM }}$ & \\
up_fom & Two-field & $\overline{\text { FOM }}$ & \\
sup_fom & Three-field & $\overline{\text { FOM }}$ & \\
irr_r9e5 & Irreducible & $\overline{\text { ROM }}$ & \\
irr_r9e6 & Irreducible & $\overline{\text { ROM }}$ & 99.999 \\
up_r9e4 & Two-field & $\overline{\text { ROM }}$ & 9999 \\
up_r9e5 & Two-field & $\overline{\text { ROM }}$ & 99.99 \\
up_r9e6 & Two-field & $\overline{\text { ROM }}$ & 99.999 \\
sup_r9e3 & Three-field & $\overline{\text { ROM }}$ & 99.99 \\
sup_r9e4 & Three-field & $\overline{\text { ROM }}$ & 99.99 \\
\hline
\end{tabular}

The FE mesh employed consists of 2278 linear elements in all cases and 1290 nodal points. Thus, the number of degrees of freedom is different for the three formulations, although we know from previous work (see [29]) than the increase in the number of degrees of freedom of the three-field formulation pays-off, i.e., it shows more accurate results for the same number of degrees of freedom. Here we only wish to compare the three formulations for a given mesh. The time step employed in all cases is $\delta t=0.001$. 
Figs. 2 and 3 show the displacement and acceleration at the tip of the beam, respectively. In these results only a portion of the transient is shown to see the behavior of each formulation. It is instructive to analyze these results because it is seen that all formulations behave in a very similar way. The three-field is the most precise as was previously shown in [29], and the two-field falls in the middle between the irreducible and three-field, as was also expected. The results labeled 'fine_mesh' were taken with a very fine mesh with quadratic elements for the irreducible formulation; upon mesh refinement all formulations converge to this results.

The next series of results show the behaviour of each of the formulations compared to their respective ROM counterparts, each one with different amount of energy. The purpose of this set of results is to validate the FOM ROM behavior to be able to confidently give the step to full FSI FOM ROM. Note that all ROM results reproduce its FOM counterpart fairly well. The irreducible FOM ROM], shown in Fig. 4, is the formulation that requires the highest amount of energy, above $99.999 \%$, to show comparable precision with its two other counterparts. The twofield, shown in Fig. 5, is as expected in between the one-field and the three field, with a minimum energy requirement of $99.99 \%$. Finally the three-field, shown in Fig. 6 requires only $99.9 \%$ of energy to obtain ROM results comparable to the FOM ones.

It is worthwhile to analyze the contour plots shown in Fig. 7, as they show the large difference between the basis of each of the formulations. One key aspect is noting that while not much can be said for the basis of the irreducible formulation, shown in Fig. 7a, and the two-field, shown in Fig. 7b, each of the modes are clearly apparent in the basis of the three-field problem, shown in Fig. 7c, this points to the more successful reduction of the problem with the three-field approach, conclusion that is supported by the results.

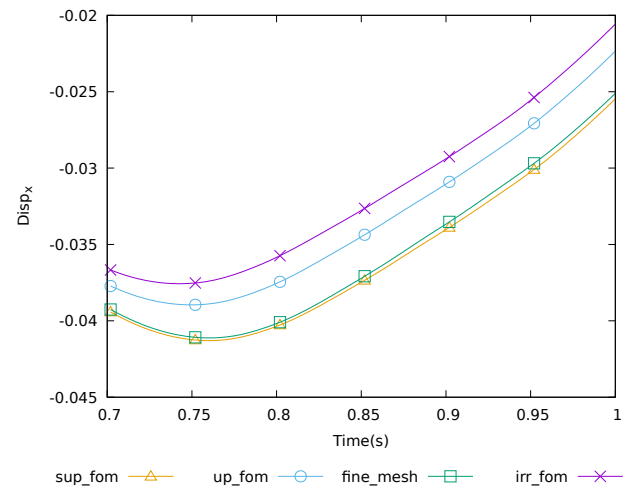

(a) Displacement in $x$ axis

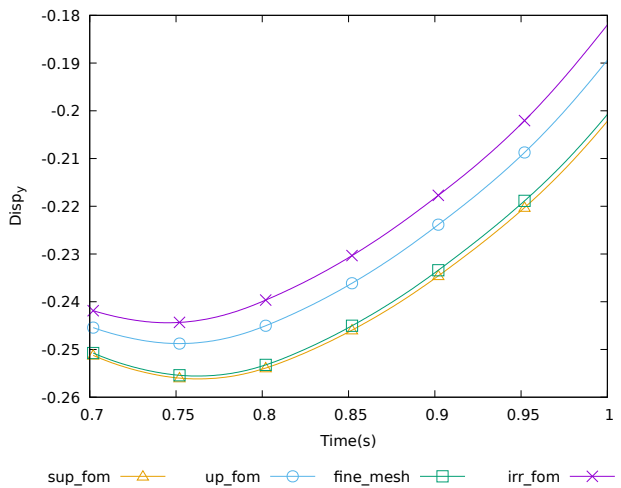

(b) Displacement in $y$ axis

Figure 2. Displacement at the tip of the beam

\subsection{Flow injection into a channel with an elastic solid}

This is a fully dynamic problem that consists of a flow injection into a bifurcating $2 \mathrm{D}$ channel with an elastic solid at the bifurcation that acts as a sort of valve. Once the fluid starts interacting with the solid, the latter starts to oscillate, expand and contract, thus changing the direction of the flow into one of the two outlets available. The geometry of the test, mesh and boundary conditions are shown in Fig. 8, notice how in the outlets elongated elements were assigned to prevent back-flow and a smooth exit for the fluid. A horizontal velocity $v_{x}=0.75$ is prescribed on the middle one third of the left wall, whereas on the rest of this wall the velocity is fixed to zero.

The test conditions are shown in Table III. where $\nu_{\mathrm{fl}}=\mu_{\mathrm{ff}} / \rho_{\mathrm{fl}}$ the kinematic viscosity of the fluid. 


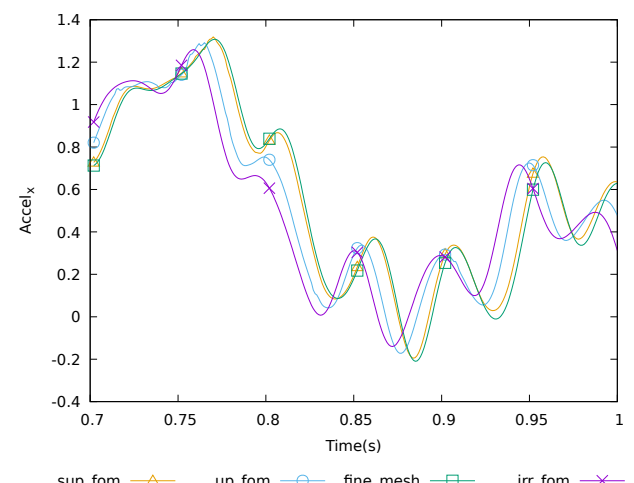

(a) Acceleration in $x$ axis

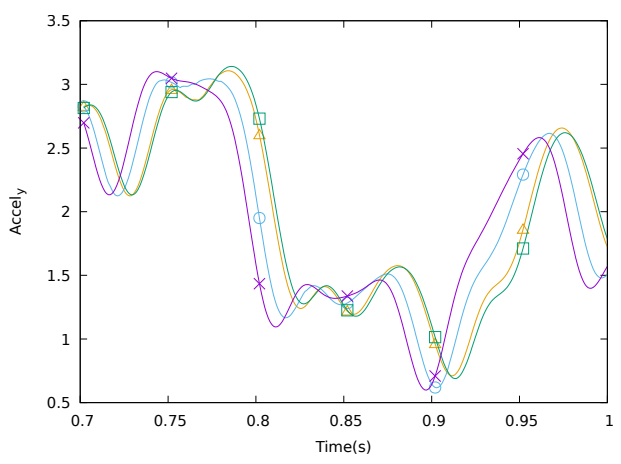

sup_fom $\triangle \quad$ up_fom $-\circ$ fine_mesh $\square$ irr_fom $\longrightarrow$

(b) Acceleration in $y$ axis

Figure 3. Acceleration at the tip of the beam

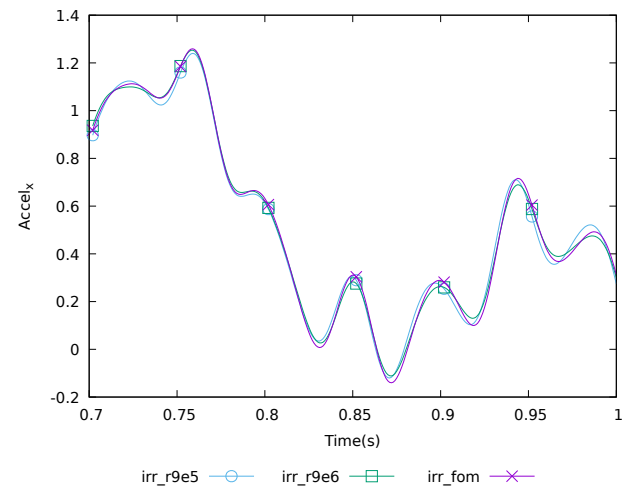

(a) Acceleration in $x$ axis

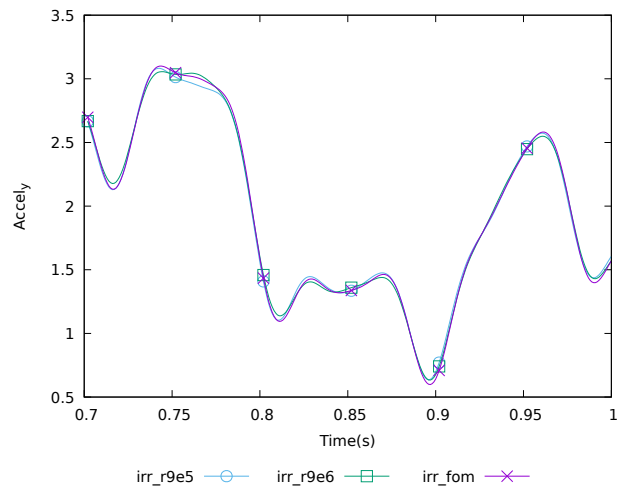

(b) Acceleration in $y$ axis

Figure 4. Acceleration at the tip of the beam, FOM-ROM irreducible

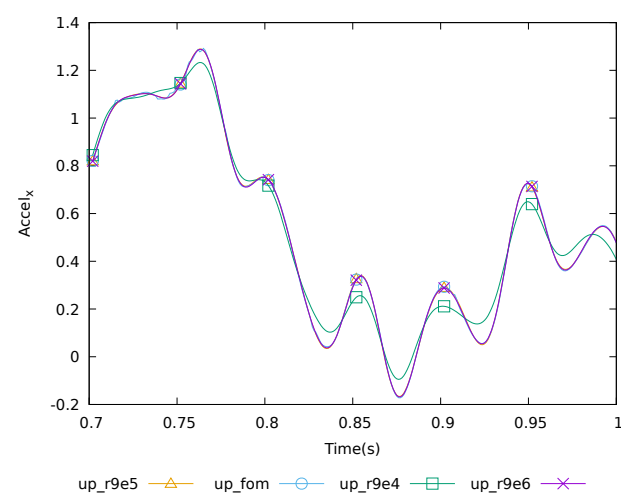

(a) Acceleration in $x$ axis

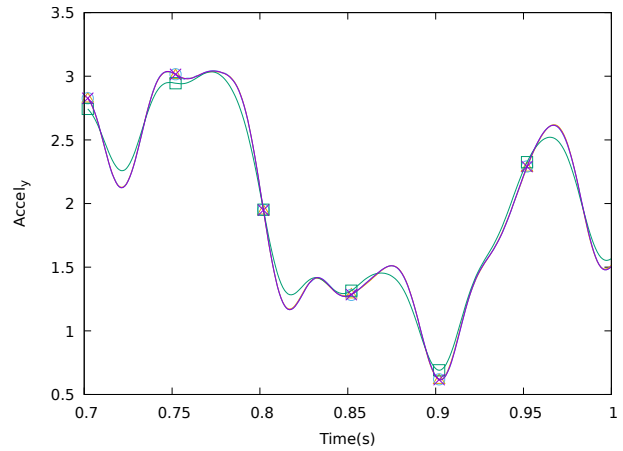

up_r9e5 $\triangle$ up_fom - up_r9e4 $\square$ up_r9e6 $\rightarrow$

(b) Acceleration in $y$ axis

Figure 5. Acceleration at the tip of the beam, FOM-ROM, two fields (UP)

Triangular quadratic elements have been used for both the fluid and the solid, with 6107 elements and 12848 nodes for the former and 3072 elements and 6323 nodes for the latter. The time step size has been taken $\delta t=0.2$. 


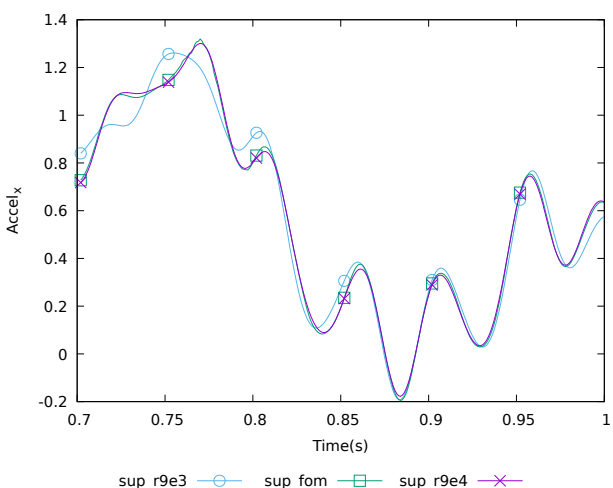

(a) Acceleration in $x$ axis

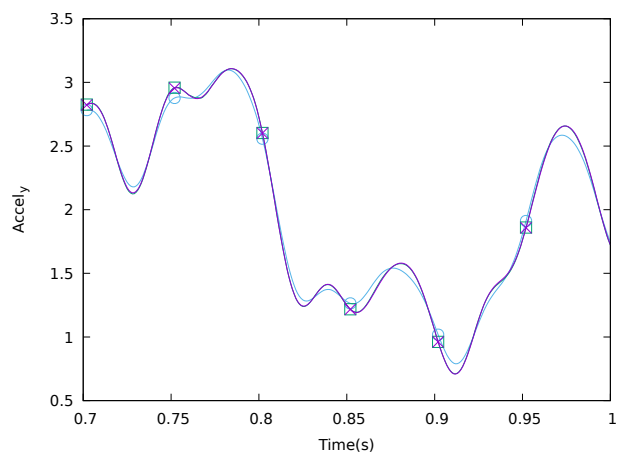

(b) Acceleration in $y$ axis

Figure 6. Acceleration at the tip of the beam, FOM-ROM, three fields (SUP)

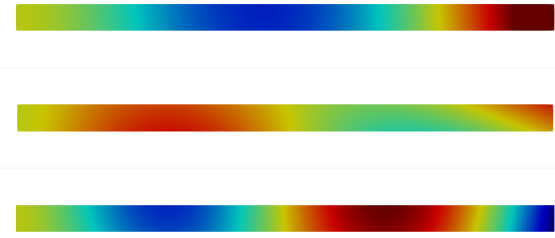

(a) IRR

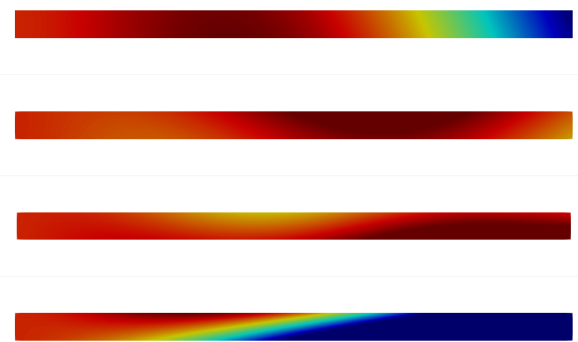

(b) UP

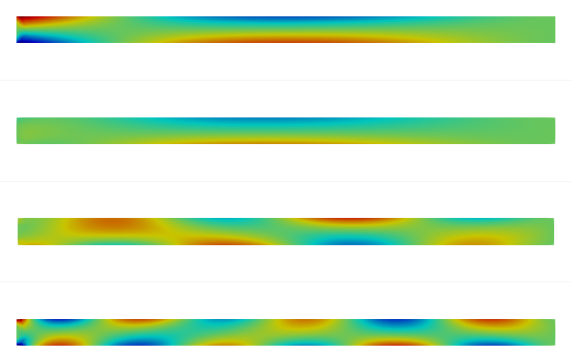

(c) SUP

Figure 7. Basis contours for the displacement in $y$ for IRR, UP and SUP

Table III. Physical parameters (SI units)

\begin{tabular}{cccc}
\hline & Fluid & & Solid \\
\hline$\rho_{\mathrm{fl}}$ & 500.0 & $\rho_{\mathrm{sl}}$ & $2,500.0$ \\
$\nu_{\mathrm{fl}}$ & 0.1 & $\nu_{\mathrm{sl}}$ & 0.3 \\
& & Young & $2,000.0$ \\
model & Newtonian & & Neo-Hookean \\
\hline
\end{tabular}

We first show the behavior of the FOM results for each formulation. As it is now expected, the both irreducible and the two-field solutions are very similar to each other, while the three-field 


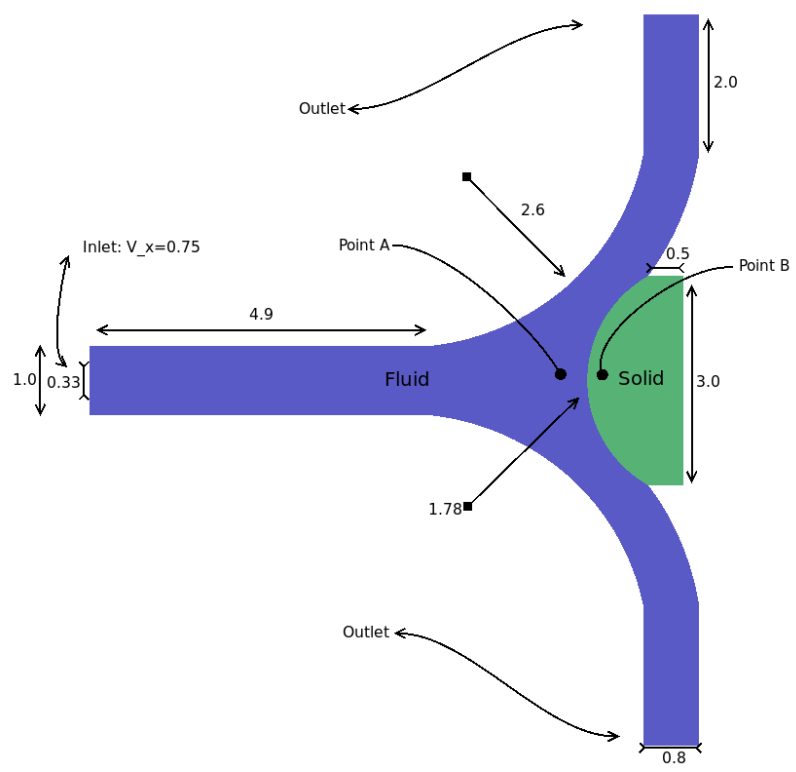

(a) Geometry for test

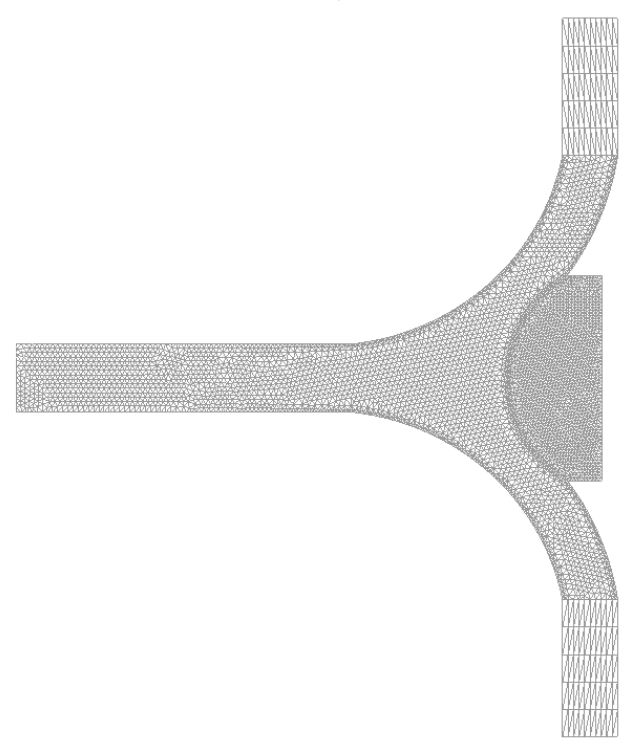

(b) Mesh used in test

Figure 8. Geometry and mesh used for fully dynamic FSI-ROM case

formulation follows a similar trend but having a higher frequency response both for pressure and velocity, see Figs. 9, 10, 11 .

Referring now to the ROM, convergence tests were done for different amounts of energy for all three formulations and the RMSD calculated for all samples. Results are shown in the following plots. Fig. 12 shows the correlation between energy and number of basis vectors for all three formulations. For the solid, the curves shown in Fig. 12a are significantly different for the three formulations. The plot for the fluid, shown on Fig. 12b, indicates that all three formulations behave similarly in terms of energy use and the number of associated basis vectors, linearly except for the highest amount of energy. Remember that for the fluid the $1 \mathrm{~F}$ and $2 \mathrm{~F}$ couplings use the same velocity-pressure formulation. 


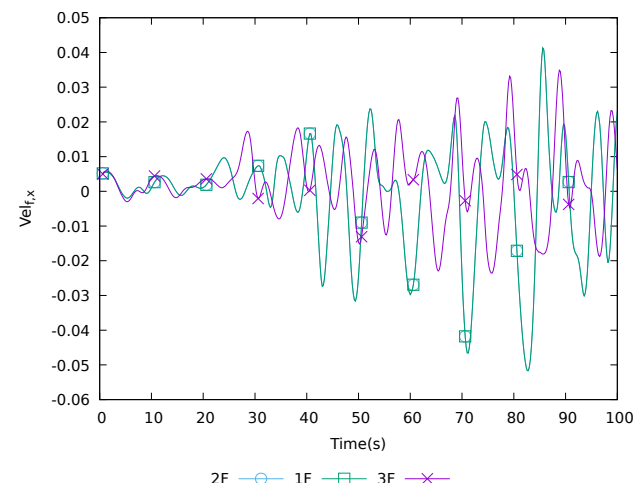

(a) Velocity in $x$

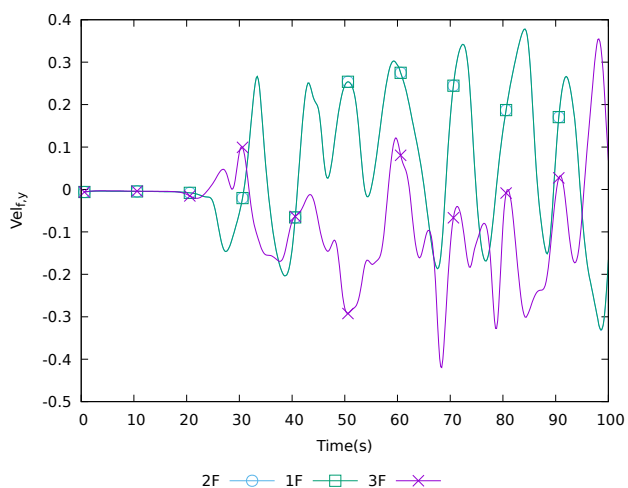

(b) Velocity in $y$

Figure 9. Velocity for the fluid at point A, FOM

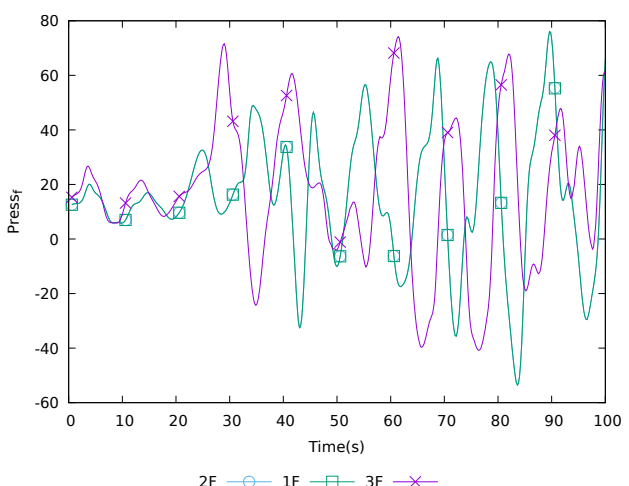

Figure 10. Pressure for the fluid at point A, FOM

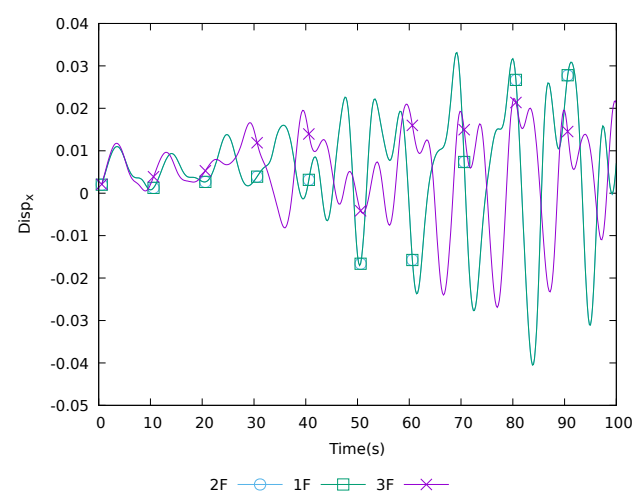

(a) Displacement in $x$

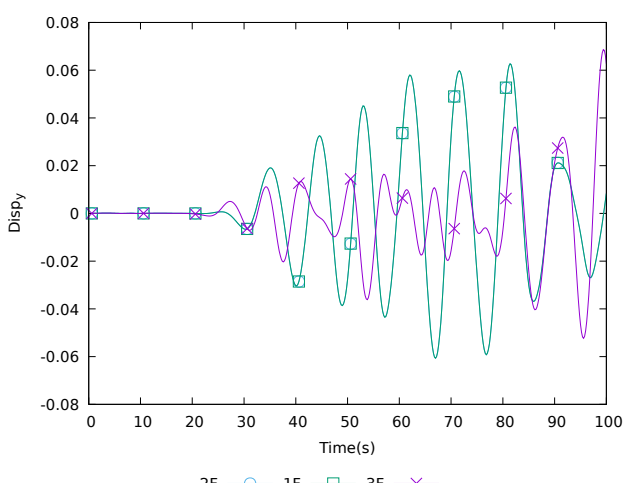

(b) Displacement in $y$

Figure 11. Displacement for the fluid at point B, FOM

Figs. 13, 14, and 15 show the evolution of the error in terms of energy and number of basis vectors. The best approximation for the displacement in the solid is obtained with the three-field formulation. The velocity in the fluid appears to be better approximated by the three-field formulation while the pressure is better approximated by the two-field approach for small values of $r$, although the slope 


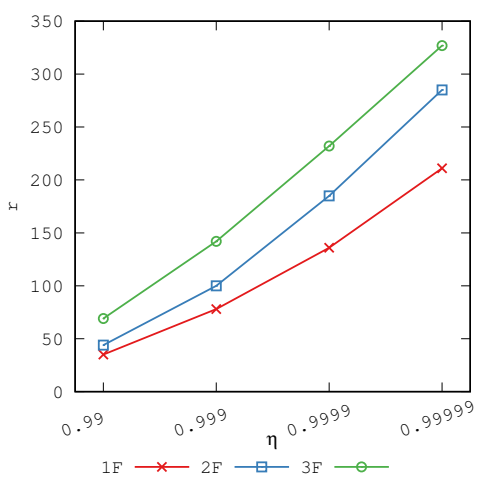

(a) Solid

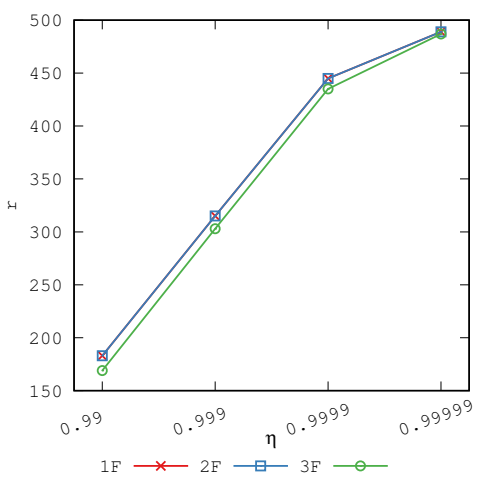

(b) Fluid

Figure 12. \# basis vectors per energy $\%$

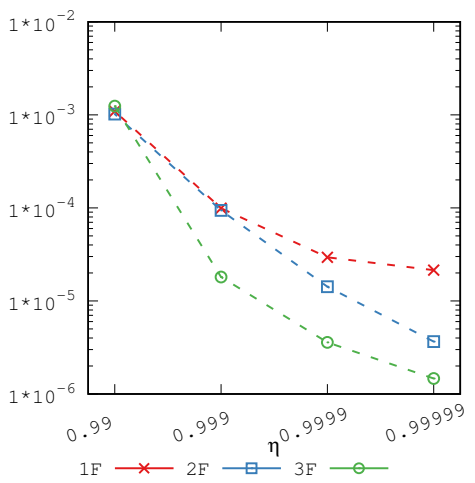

(a) Energy \%

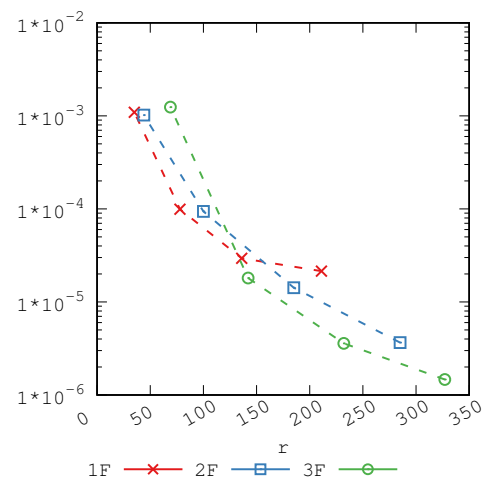

(b) \# Basis vectors

Figure 13. Convergence for ROM displacement in the solid at point B per basis vectors and energy

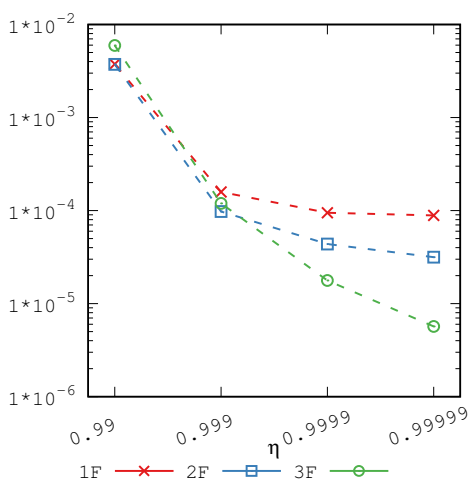

(a) Energy \%

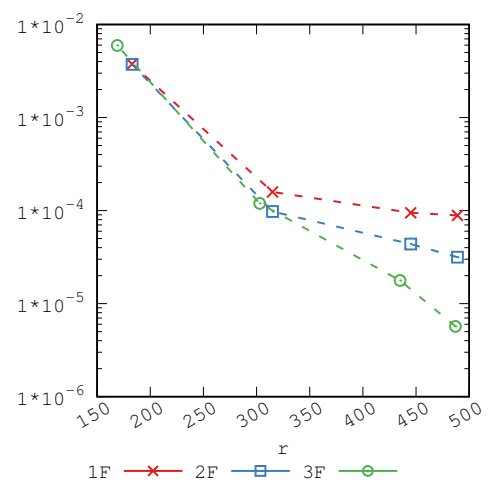

(b) \# Basis vectors

Figure 14. Convergence for ROM velocity in the fluid at point A per basis vectors and energy

of the three-field formulation seems to be higher. In general the $\mathrm{F} 2 \mathrm{~F}$ coupling is more precise than the standard coupling.

Good results have been obtained for the approximation of this problem by means of all formulations, but the most precise is the three-field one. It is illustrative to see how the response of the ROM correlates with the FOM. In this order of ideas, graphs of the dynamic response are shown for both 


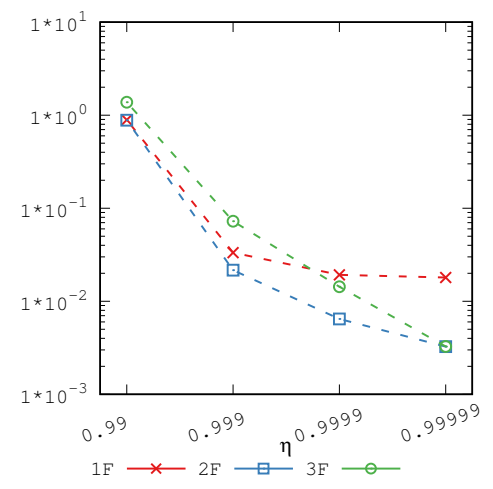

(a) Energy \%

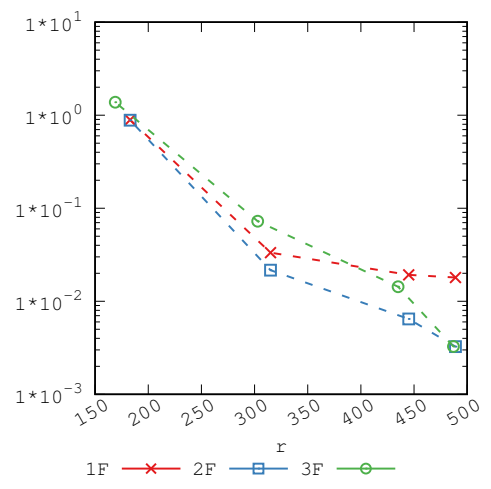

(b) \# Basis vectors

Figure 15. Convergence for ROM pressure in the fluid at point A per basis vectors and energy

FOM and ROM comparing different amounts of energy for the three-field scenario. Table IV] shows the explanation for the legend in the graphs.

Table IV. Case parameters

\begin{tabular}{ccc|c}
\hline Name & Formulation & \multicolumn{1}{l}{ Space } & Energy(\%) \\
\hline fom & Three-field & FOM & \\
rom_99 & Three-field & $\overline{\text { ROM }}$ & 99.0 \\
rom_9e3 & Three-field & $\overline{\text { ROM }}$ & 99.9 \\
rom_9e4 & Three-field & $\overline{\text { ROM }}$ & 99.99 \\
rom_9e5 & Three-field & $\overline{\text { ROM }}$ & 99.999 \\
\hline
\end{tabular}

Figs. 16 and 17 show the displacement of the solid at point $\mathrm{B}$ and velocity for the fluid at point A, respectively. Notice that the dynamic response is quite complex, but nevertheless, it is correctly captured by all ROM cases. There is a slight overshoot for the amplitude response for rom_99, but phase is preserved.

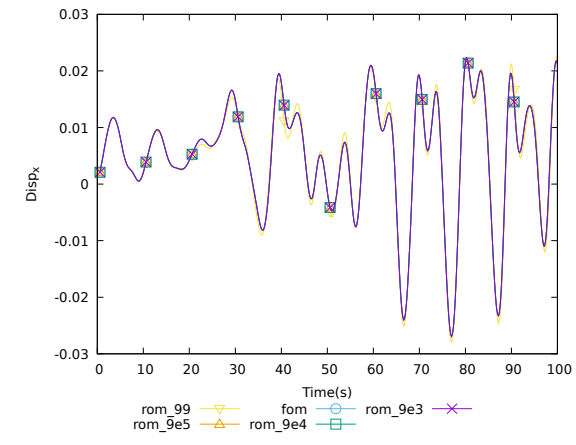

(a) Displacement $x$

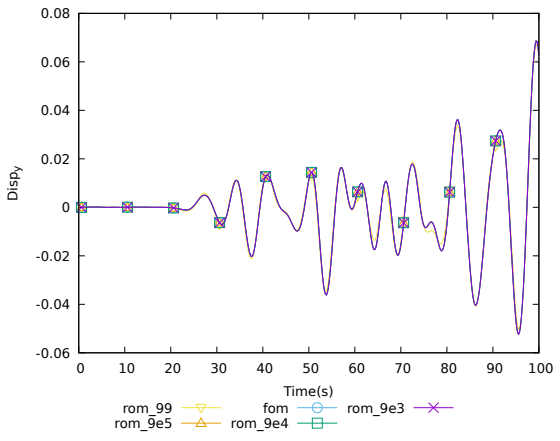

(b) Displacement $y$

Figure 16. FOM-ROM for solid displacement at point B for the three-field formulation

Lastly, to exemplify the complexity of the flow, contour plots for some variables are shown next for different intervals of the dynamic evolution. Notice how the flow starts, develops, starts affecting the solid and finally a feedback loop is started from the coupling. All contour plots are taken from the rom_9e5 results. 


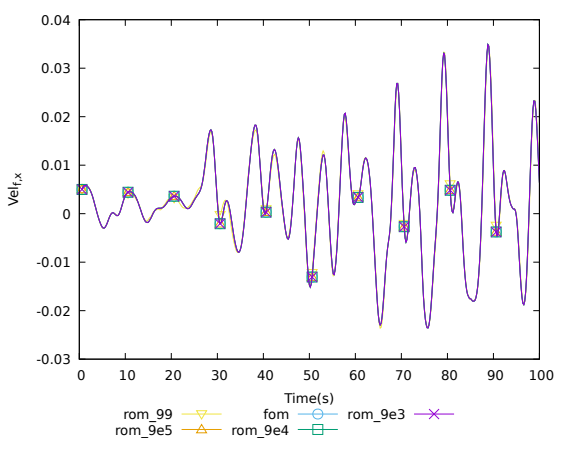

(a) Velocity $x$

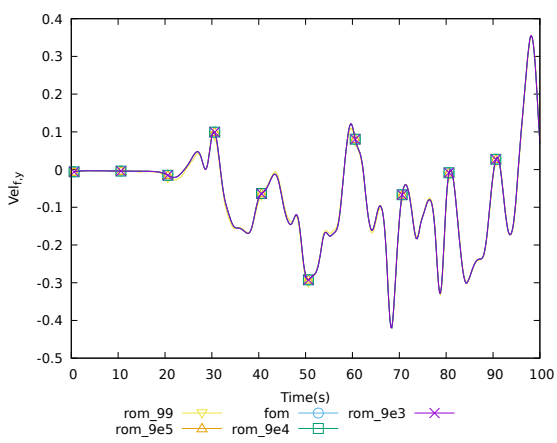

(b) Velocity $y$

Figure 17. FOM-ROM for fluid velocity at point A for the three-field formulation

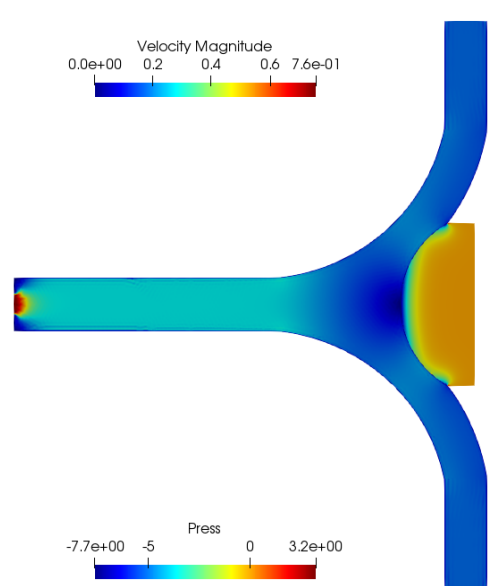

(a) Fluid velocity and solid pressure

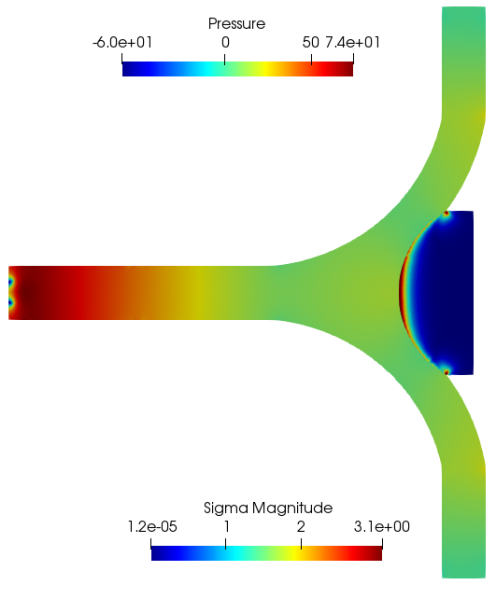

(b) Fluid pressure and solid deviatoric stress

Figure 18. Fluid and solid solution contours at $t=0 \mathrm{~s}$

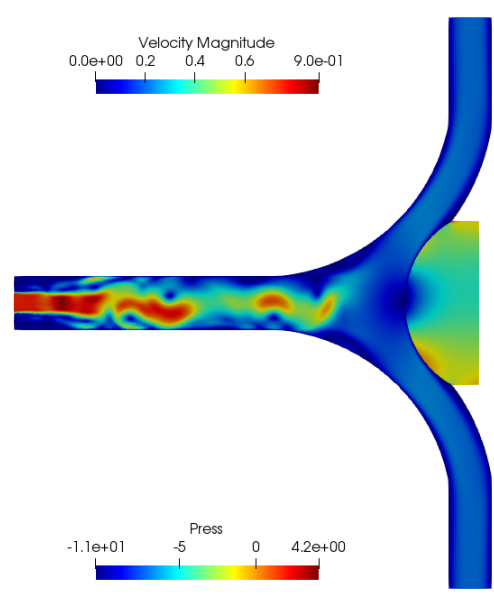

(a) Fluid velocity and solid pressure

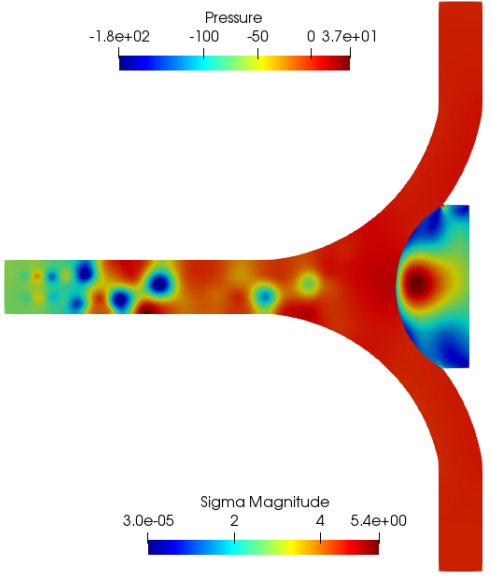

(b) Fluid pressure and solid deviatoric stress

Figure 19. Fluid and Solid solution contours at $t=20 \mathrm{~s}$ 


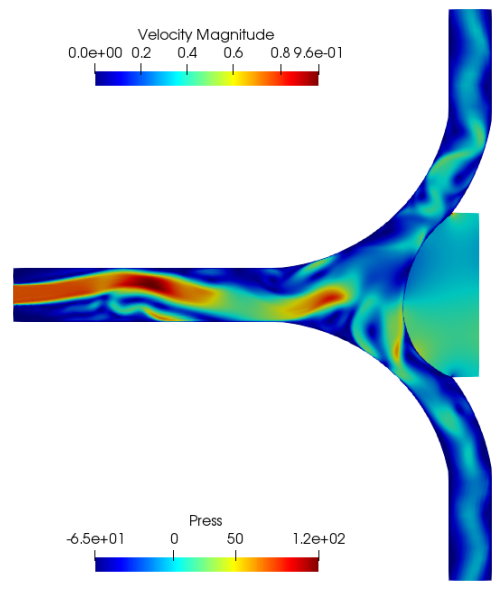

(a) Fluid velocity and solid pressure

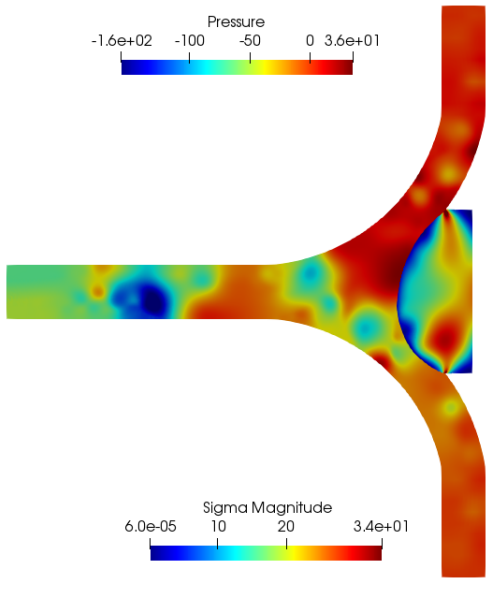

(b) Fluid pressure and solid deviatoric stress

Figure 20. Fluid and solid solution contours for $t=52 \mathrm{~s}$

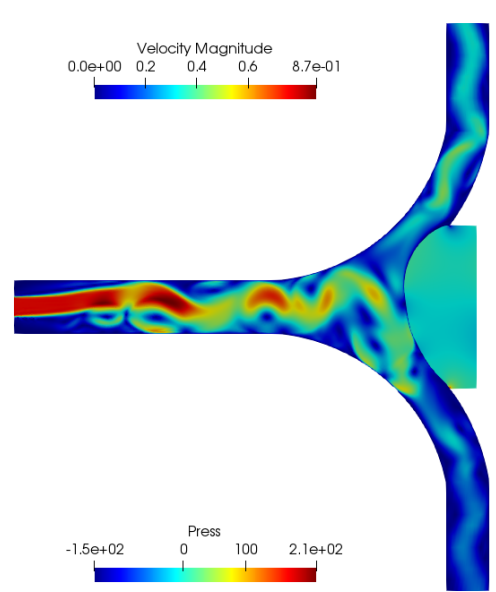

(a) Fluid velocity and solid pressure

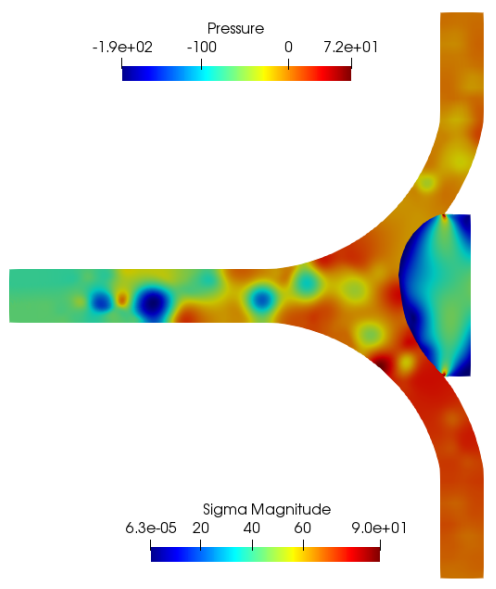

(b) Fluid pressure and solid deviatoric stress

Figure 21. Fluid and solid solution contours for $t=90 \mathrm{~s}$

Overall this test shows a complex scenario with a challenging dynamic response that was correctly captured by all formulations, the most precise being the $\mathrm{F} 2 \mathrm{~F}$ couplings.

\subsection{D Flow around a plate}

This last example consists of a flow over a 3D plate. The test conditions are shown in Table $\mathrm{V}$. The geometry is shown in Fig. 22 and Table VI shows important mesh parameters; geometrical parameters are shown in Table VII and Table VIII shows the boundary conditions.

For this example only the results for the three-field $\mathrm{FSI}$ are shown as from previous results they are expected to be the most precise. These results are shown as a qualitative example that good approximation can even be obtained for very coarse meshes with the three-field coupling. Results are shown as a comparison for the FOM and a series of ROM cases ran with varying amount of energy. The legend for the results can be found in Table IX. Accurate results have been obtained for all variable shown. It is seen that the solid tends to a stationary configuration; in spite of a 
Table V. Physical parameters (SI units)

\begin{tabular}{cccc}
\hline & Fluid & & Solid \\
\hline$\rho_{\mathrm{fl}}$ & 100.0 & $\rho_{\mathrm{sl}}$ & $1,000.0$ \\
$\nu_{\mathrm{fl}}$ & 1.0 & $\nu_{\mathrm{sl}}$ & 0.48 \\
& & Young & $300 \times 10^{3}$ \\
model & Newtonian & & NeoHookean \\
\hline
\end{tabular}

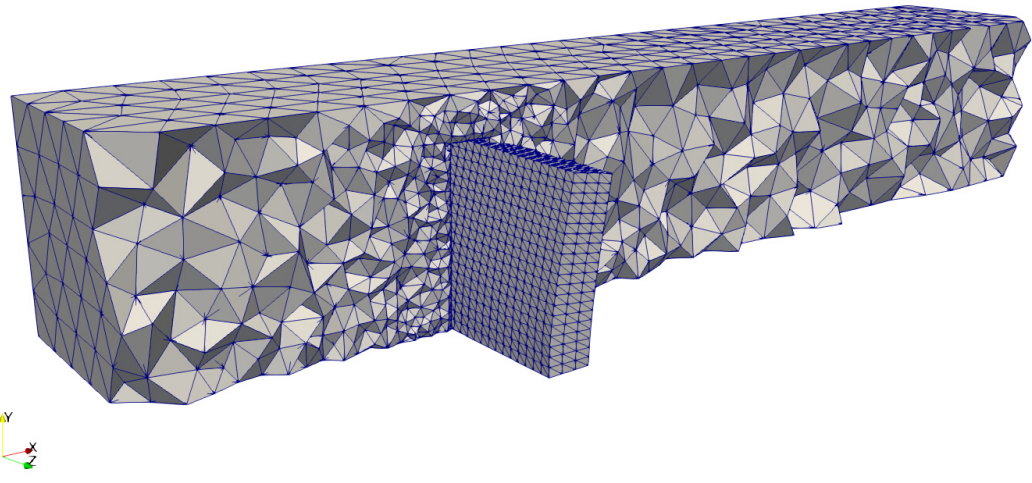

Figure 22. Case geometry

Table VI. Mesh parameters

\begin{tabular}{ccc}
\hline & Fluid & Solid \\
\hline Element type & Linear Tetrahedra & Linear Tetrahedra \\
Nodes per element & 4 & 4 \\
\# of elements & 87,941 & 10,833 \\
\# of nodes & 16,785 & 2,491 \\
\hline
\end{tabular}

Table VII. Channel dimensions and flow parameters

\begin{tabular}{cc}
\hline Height $(\mathrm{H}):$ & 0.5 \\
Width : & 1.0 \\
Length: & 3.0 \\
Inlet mean velocity $(\bar{U}):$ & 1.0
\end{tabular}

Table VIII. Boundary conditions

\begin{tabular}{ccc}
\hline & Fluid & Solid \\
\hline Flow inlet: & $1.5 \bar{U} \cdot \frac{y \cdot(0.5-y) \cdot z \cdot(1.0-z)}{\left(\frac{H}{2}\right)^{2}}$ & \\
Flow outlet: & Free & \\
Channel walls: & No slip & \\
Plate sides: & Solid velocities & Fluid tractions \\
Plate bottom: & & Fixed \\
\hline
\end{tabular}

slightly different transient for all formulations considered, this steady state is the same for all approaches. 
Table IX. Case parameters

\begin{tabular}{ccc|c}
\hline Name & Formulation & Space & Energy(\%) \\
\hline fom & Three-field & FOM & \\
rom_e99 & Three-field & $\overline{\text { ROM }}$ & 99.0 \\
rom_e9e3 & Three-field & $\overline{\text { ROM }}$ & 99.9 \\
rom_e9e4 & Three-field & $\overline{\text { ROM }}$ & 99.99 \\
rom_e9e5 & Three-field & $\overline{\text { ROM }}$ & 99.999 \\
\hline
\end{tabular}

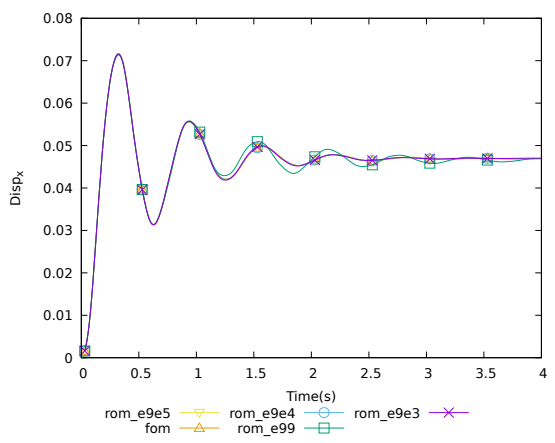

(a) Displacement in $x$

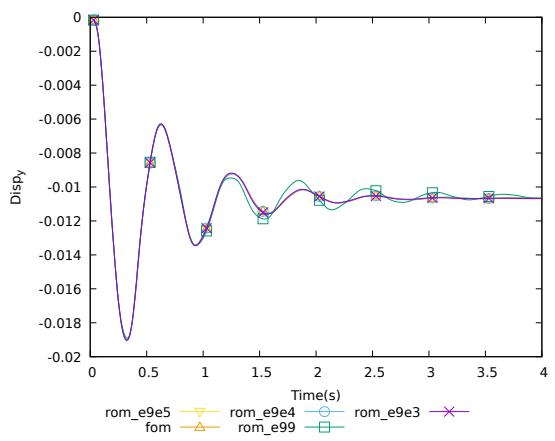

(b) Displacement in $y$

Figure 23. Displacement at the tip of the plate

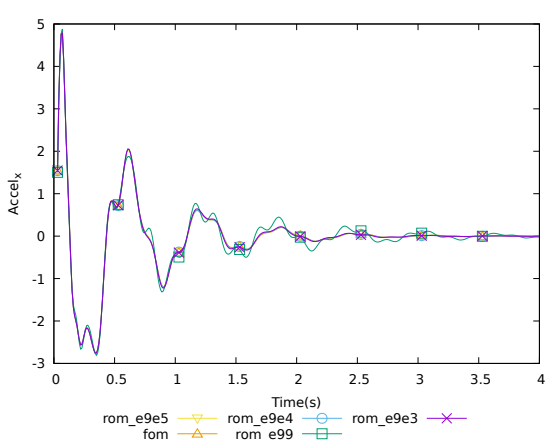

(a) Acceleration in $x$

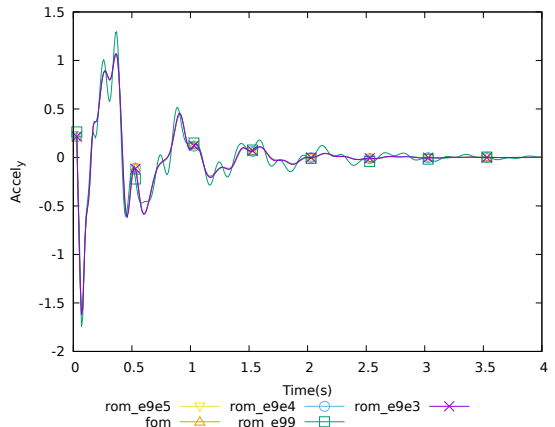

(b) Acceleration in $y$

Figure 24. Acceleration at the tip of the plate

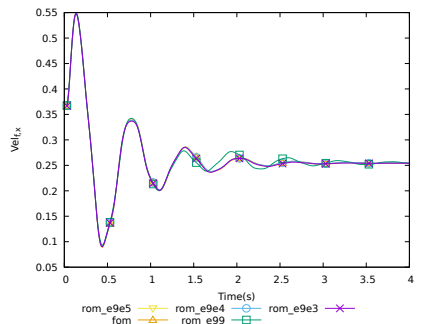

(a) Velocity in $x$

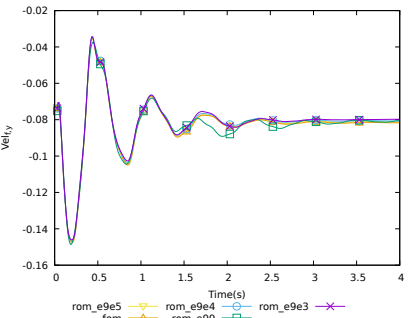

(b) Velocity in $y$

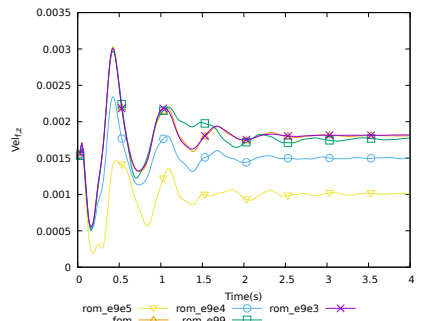

(c) Velocity in $z$

Figure 25. Velocity for the fluid at the tip of the plate 


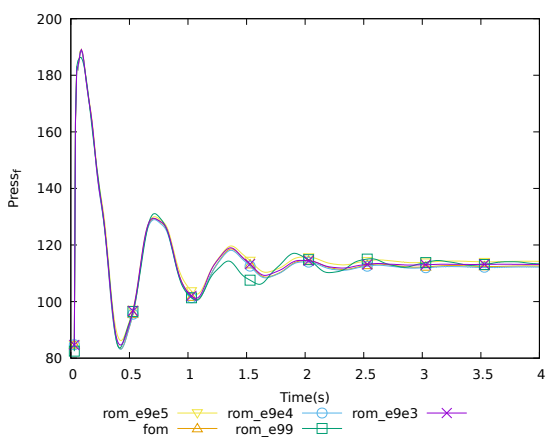

(a) Fluid

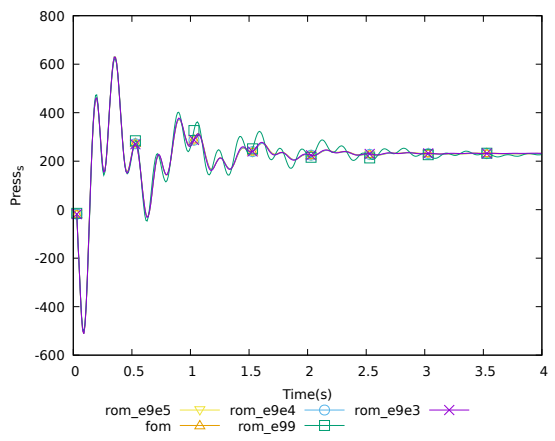

(b) Solid

Figure 26. Pressure for fluid and solid at the tip of the plate

\section{GENERAL CONCLUSIONS AND OUTLOOK}

A two-field elasto-dynamic formulation has been introduced with the objective of completing our F2F paired FSI models in order to compare three different types of coupling and their advantages. The two-field formulation proves to be more precise than the irreducible formulation with just one more degree of freedom, but less so than its three-field counterpart. In fact in every test the two-field was in between the irreducible and three-field formulations.

Convergence tests were done for FSI ROM cases where it was seen that in the ROM space there is a threshold where a reduced problem will give meaningful results; for smaller ROM spaces the solution will not be precise enough. As it is well known and in fact we have observed, for much larger ROM spaces the solution may be polluted by the effects of overfitting and can differ from the FOM This upper threshold, though problem dependent, tends to be reduced for the irreducible formulation, larger for the two-field and quite large for the three-field (not even visible in our experience), making the latter the most efficient as it counts with a wide range of applicability before overfitting starts to affect the solution.

The performance, both in FOM and ROM spaces but specially in the latter, of the two-field FSI was shown to be superior to the standard coupling, even though both use the same velocitypressure formulation for the fluid. The gain in performance appears to be not from the type for formulation used, but rather from the type of coupling, making $[\mathrm{F} 2 \mathrm{~F}$ coupling worthwhile whenever possible.

The three-field formulation produces in the fluid a richer basis with higher magnitude than its counterparts. In the solid it produces a basis with marked modes. This hints at a better reduction of the problem achieved by a correct splitting of the stress tensor, which in turn produces a more accurate reduced problem.

Overall the three-field formulation, both for the solid and for the $\mathrm{F} 2 \mathrm{~F}$ coupling, proves to be superior, albeit more expensive, both in the FOM and ROM spaces in terms of accuracy. In any case given that this formulation has been shown to be very resilient to time step, mesh and number of basis used, it still remains competitive with regard to the other types of couplings. It was also seen that a mixed field coupling involving any three-field formulation will be more precise than its counterparts, the best one being the one with a three-field formulation in the solid. This is also convenient as generally the fluid is the one with the largest number of degrees of freedom. If a $2 \mathrm{~F} / 3 \mathrm{~F}$ provides accurate results and computational cost is of concern, then it should be preferred to its expensive counterpart, the full three-field coupling. 
It is intuitive to assume that given that there are three versions of the FSI problem both in FOM and ROM it is possible to apply the calculated basis from one problem to another. This idea can have various advantages which are:

- Basis calculated in the more accurate three-field problem applied to the standard problem. The idea in reduced order modeling is to run the most precise offline stage possible so the calculated basis can reproduce the original problem faithfully. In this way it makes sense to calculate it with the three-field approach, and then apply it to an online stage that is focused on performance, this is, it is the fastest possible, for which it makes sense to apply the standard coupling.

- Basis calculated in the less accurate standard problem applied to the more accurate threefield problem. There are real world applications in which the geometry dictates the size of the mesh to be used. Small geometric detaisl can force a very high number of nodes. In this way running a FOM with the three-field coupling can become unfeasible as calculation times become very high. The problem can be solved by means of the standard coupling and the online stage run with a more precise three-field approach that guarantees precision in the ROM stage.

Unfortunately, in our experience neither of the two approaches worked readily, meaning that some degree of processing and manipulation should be done to each basis so that it can be applied to another formulation of the FSI problem. This idea is left as future work.

\section{ACKNOWLEDGEMENTS}

A. Tello wants to acknowledge the doctoral scholarship received from the Colombian GovernmentColciencias. R. Codina gratefully acknowledges the support received from the ICREA Acadèmia Program, from the Catalan Government.

\section{REFERENCES}

1. J. Baiges, R. Codina, and S. Idelsohn. Reduced-order subscales for POD models. Computer Methods in Applied Mechanics and Engineering, 291:173-196, 72015.

2. F. Ballarin and G. Rozza. POD-Galerkin monolithic reduced order models for parametrized fluid-structure interaction problems. International Journal for Numerical Methods in Fluids, 82(12):1010-1034, 2016.

3. F. Ballarin, G. Rozza, and Y. Maday. Reduced-Order Semi-Implicit Schemes for Fluid-Structure Interaction Problems, in Model Reduction of Parametrized Systems, Benner P., Ohlberger M., Patera A., Rozza G., Urban K. (eds), pages 149 - 167. MS\&A (Modeling, Simulation and Applications), vol 17. Springer, Cham, 2017.

4. K. Carlberg, C. Bou-Mosleh, and C. Farhat. Efficient non-linear model reduction via a least-squares PetrovGalerkin projection and compressive tensor approximations. International Journal for Numerical Methods in Engineering, 86(2):155-181, 42011.

5. K. Carlberg, C. Farhat, J. Cortial, and D. Amsallem. The GNAT method for nonlinear model reduction: Effective implementation and application to computational fluid dynamics and turbulent flows. Journal of Computational Physics, 242:623-647, 2013.

6. E. Castillo and R. Codina. Stabilized stress-velocity-pressure finite element formulations of the Navier-Stokes problem for fluids with non-linear viscosity. Computer Methods in Applied Mechanics and Engineering, 279:554578, 92014.

7. G. Chiandussi, G. Bugeda, and E. Oñate. A simple method for automatic update of finite element meshes. Communications in Numerical Methods in Engineering, 16(1):1-19, 12000.

8. R. Codina. Stabilized finite element approximation of transient incompressible flows using orthogonal subscales. Computer Methods in Applied Mechanics and Engineering, 191(39-40):4295-4321, 82002.

9. R. Codina. Finite Element Approximation of the Three-Field Formulation of the Stokes Problem Using Arbitrary Interpolations. SIAM Journal on Numerical Analysis, 47(1):699-718, 12009.

10. R. Codina, S. Badia, J. Baiges, and J. Principe. Variational multiscale methods in computational fluid dynamics. Encyclopedia of Computational Mechanics Second Edition, pages 1-28, 2018.

11. R. Codina, J. Principe, and J. Baiges. Subscales on the element boundaries in the variational two-scale finite element method. Computer Methods in Applied Mechanics and Engineering, 198(5-8):838-852, 2009. 
12. R. Codina, J. Principe, O. Guasch, and S. Badia. Time dependent subscales in the stabilized finite element approximation of incompressible flow problems. Computer Methods in Applied Mechanics and Engineering, 196(21-24):2413-2430, 42007.

13. J. Donea, A. Huerta, J. P. Ponthot, and A. Rodriguez-Ferran. Arbitrary Lagrangian-Eulerian Methods. In Encyclopedia of Computational Mechanics, pages 1-25. John Wiley \& Sons, Ltd, Chichester, UK, 112004.

14. G. Houzeaux and R. Codina. Transmission conditions with constraints in finite element domain decomposition methods for flow problems. Communications in Numerical Methods in Engineering, 17(3):179-190, 22001.

15. T. J. R. Hughes, G. R. Feijóo, L. Mazzei, and J.-B. Quincy. The variational multiscale method-a paradigm for computational mechanics. Computer Methods in Applied Mechanics and Engineering, 166(1-2):3-24, 1998.

16. T. Iliescu and Z. Wang. Variational multiscale proper orthogonal decomposition: Navier-stokes equations. Numerical Methods for Partial Differential Equations, 30(2):641-663, 32014.

17. B. M. Irons and R. C. Tuck. A version of the Aitken accelerator for computer iteration. International Journal for Numerical Methods in Engineering, 1(3):275-277, 71969.

18. I. Kalashnikova, M. Barone, and M. Brake. A stable Galerkin reduced order model for coupled fluid-structure interaction problems. International Journal for Numerical Methods in Engineering, 95(2):121-144, 2013.

19. U. Küttler and W. A. Wall. Fixed-point fluid-structure interaction solvers with dynamic relaxation. Computational Mechanics, 43(1):61-72, 2008.

20. Z. Li, Y. Chen, S. Chang, and H. Luo. A Reduced-Order Flow Model for Fluid-Structure Interaction Simulation of Vocal Fold Vibration. Journal of Biomechanical Engineering, 142(2), 10 2019. 021005.

21. T. Lieu, C. Farhat, and M. Lesoinne. Reduced-order fluid/structure modeling of a complete aircraft configuration. Computer Methods in Applied Mechanics and Engineering, 195(41):5730 - 5742, 2006. John H. Argyris Memorial Issue. Part II.

22. R. Reyes and R. Codina. Element boundary terms in reduced order models for flow problems: domain decomposition and adaptive coarse mesh. Submitted, pages 1-32, 2019.

23. R. Reyes and R. Codina. Projection-based reduced order models for flow problems: A variational multiscale approach. Computer Methods in Applied Mechanics and Engineering, To appear.

24. R. Reyes, R. Codina, J. Baiges, and S. Idelsohn. Reduced order models for thermally coupled low Mach flows. Advanced Modeling and Simulation in Engineering Sciences, 5(1):28, 122018.

25. V. Ruas. Une methode mixte contrainte-deplacement-pression pour la resolution de problemes de viscoelasticite incompressible en deformations planes. Comptes rendus de l'Acad $\backslash$ 'emie des Sciences. $S \backslash$ 'erie 2, 301:1171-1174, 1985.

26. D. Ryckelynck. A priori hyperreduction method: an adaptive approach. Journal of Computational Physics, 202(1):346-366, 12005

27. L. Sirovich and M. Kirby. Low-dimensional procedure for the characterization of human faces. Journal of the Optical Society of America A, 4(3):519, 31987.

28. G. Stabile, F. Ballarin, G. Zuccarino, and G. Rozza. A reduced order variational multiscale approach for turbulent flows. Advances in Computational Mathematics, 112019.

29. A. Tello and R. Codina. Three Field Fluid Structure Interaction by means of the Variational Multiscale method. Submitted, 2020.

30. A. Tello, R. Codina, and J. Baiges. Fluid Structure Interaction by means of Variational Multiscale Reduced Order Models. International Journal for Numerical Methods in Engineering, To Appear:nme.6321, 22020.

31. J. Vierendeels, L. Lanoye, J. Degroote, and P. Verdonck. Implicit coupling of partitioned fluid-structure interaction problems with reduced order models. Computers and Structures, 85(11-14):970-976, 2007.

32. W. A. Wall, D. P. Mok, and E. Ramm. Iterative Substructering Schemes for Fluid Structure Interaction. In Lecture Notes in Applied and Computational Mechanics, volume 12, pages 349-360. 2003.

33. W. Wang and Y. Yan. Strongly coupling of partitioned fluid-solid interaction solvers using reduced-order models. Applied Mathematical Modelling, 34(12):3817 - 3830, 2010. 